\title{
OVO: \\ UM NOVO PROBLEMA DE OTIMIZAÇÃO
}

\author{
Cibele Dunder
}

\author{
TESE APRESENTADA \\ $\mathrm{AO}$ \\ INSTITUTO DE MATEMÁTICA E ESTATÍSTICA \\ DA \\ UNIVERSIDADE DE SÃO PAULO \\ PARA OBTENÇÃO DO GRAU DE DOUTOR \\ EM \\ MATEMÁTICA APLICADA \\ Área de Concentração: Matemática Aplicada \\ Orientador: Prof. Dr. José Mario Martínez \\ Co-Orientador: Prof. Dr. Roberto Andreani
}

Durante a elaboração deste trabalho, o autor recebeu apoio de FAPESP (proc. 98/03668-0)

- São Paulo, novembro de 2002 - 


\section{OVO: Um Novo Problema de Otimização}

Este exemplar corresponde à redação final da tese devidamente corrigida e defendida por Cibele Dunder e aprovada pela comissão julgadora.

Banca Examinadora:

- Prof. Dr. Oswaldo Luiz do Valle Costa - POLI - USP

- Profa. Dra. Ana Friedlander - IMECC - UNICAMP

- Prof. Dr. Alfredo Noel Iusem - IMPA

- Profa. Dra. Susana Scheimberg de Makler - IM UFRJ

- Prof. Dr. José Mario Martínez (Orientador) - IMECC

- UNICAMP 


\section{Agradecimentos}

Primeiramente, não posso deixar de ressaltar a maestria com que o Professor José Mario Martínez conduz suas orientações. Um talento que poucos têm ... Ao meu orientador, meus sinceros agradecimentos por toda atenção dada a este trabalho. Não menos importante está meu co-orientador, o Professor Roberto Andreani, a quem agradeço pelo constante entusiasmo, parceria e bom humor sempre.

Também não posso deixar de agradecer aos grupos de otimização do IME-USP e do IMECC-UNICAMP, que muitas vezes discutiram e deram valiosas sugestões sobre o problema OVO nos seminários.

Em especial, agradeço os Professores Carlos Humes Jr. e Júlio M. Stern, que me despertaram o interesse pela otimização e muito me incentivaram a chegar até a defesa desta tese.

A todos, 


\section{Sumário}

$\begin{array}{lll}\text { Resumo iv } & \text { iv }\end{array}$

Abstract $\quad$ v

1 Introdução 1

2 Otimização do Valor Ordenado 4

2.1 Introdução . . . . . . . . . . . . . . . . . . 4

2.2 Reformulação do problema . . . . . . . . . . . . 5

2.3 Viabilidade do problema $\mathrm{OVO}^{*} \ldots \ldots \ldots \ldots$

3 Condições de Otimalidade 14

3.1 Viabilidade de $\mathrm{OVO}^{*}$ a partir de OVO . . . . . . . . 15

3.2 Condições de otimalidade para OVO . . . . . . . . . 17

3.3 Condição de otimalidade para OVO* . . . . . . . . . . 22

3.3.1 Relacionando minimizadores de OVO e OVO* . . . 22

3.3.2 Particularidades do caso convexo . . . . . . . . . 30

3.4 Restauração e conjunto tangente . . . . . . . . . . . . 31

3.4.1 Explorando o conjunto tangente . . . . . . . . 37

3.5 Gradiente Projetado Restaurado . . . . . . . . . . . . . . 41

4 OVO e o Problema de Desigualdade Variacional 43

4.1 Introdução. . . . . . . . . . . . . . . . . . . . . . 43

$4.2 \mathrm{Um}$ resultado para VIP . . . . . . . . . . . . 45

4.3 Extensão dos resultados ao problema OVO . . . . . . . . 49

5 Método Cauchy Primal para OVO 51

5.1 Introdução . . . . . . . . . . . . . . . . . . . . 51

5.2 O algoritmo . . . . . . . . . . . . . . . 52

5.3 Calculando uma solução aproximada para o subproblema . . 59 
6 Experimentos Numéricos

61

7 Conclusões

66

Referências Bibliográficas

68 


\section{Resumo}

Definimos um novo problema problema de otimização, chamado problema de Otimização do Valor Ordenado ("Order Value Optimization" ou OVO). Este é um problema não diferenciável, não linear e, em geral, não convexo. Para lidar com estas características, reformulamos o problema OVO como um problema de programação matemática com restrições de equilíbrio. Os primeiros quatro capítulos são dedicados à análise desta equivalência. Apresentamos condições de otimalidade de primeira e segunda ordem para OVO, estabelecendo a relação entre minimizadores de OVO e sua reformulação. O quinto capítulo apresenta um algoritmo primal para o problema OVO, chamado de método do tipo Cauchy, que resolve o problema de forma que todo ponto limite satisfaz uma adequada condição de otimalidade. Alguns exemplos numéricos e aplicações do problema OVO estão no sexto capítulo. As conclusões e perspectivas futuras são dadas no capítulo 7 . 


\section{Abstract}

We define a new optimization problem, called Order Value Optimization Problem (OVO). This is a nonsmooth, nonlinear and, in general, nonconvex programming problem. To deal with these characteristics, we prove the equivalence between OVO and a mathematical programming problem with equilibrium constraints. The first four chapters are dedicated to the analysis of this equivalence. We also give some results about first and second order optimality conditions, making a connection between stationary points of OVO and its reformulation. The fifth chapter is devoted to the OVO problem in its primal form, that is, only with the original variables. A Cauchy-type method is introduced that solves the problem in the sense that every limit point satisfies an adequate optimality condition. Numerical examples and applications of OVO problem are given in chapter 6. Conclusions and future research are discussed in chapter 7 . 


\section{Capítulo 1}

\section{Introdução}

O objetivo deste trabalho é apresentar um novo problema de otimização, que é uma generalização do clássico problema Minimax. Ao invés de otimizar o máximo (ou o mínimo) de um conjunto de funções, trabalharemos com o valor funcional que representa a p-ésima menor função neste conjunto, para algum valor inteiro $p$ fixado a priori. Este novo problema, chamado "Otimização do Valor Ordenado", ou em inglês "Order Value Optimization" (OVO), é um problema de otimização contínua, não diferenciável e, em geral, não convexo. Estas características criam uma certa dificuldade em resolver computacionalmente o problema. Para driblar os obstáculos desenvolvemos duas linhas de pesquisa complementares. Na primeira linha, reformulamos matematicamente o problema e obtemos uma equivalência entre o problema OVO e um problema de programação matemática com restrições de equilíbrio (MPEC). Desta reformulação exploramos todas as propriedades da estrutura deste novo PNL e obtemos alguns resultados que nos permitem usar algoritmos já conhecidos e comprovadamente eficientes para resolver OVO. A segunda linha de pesquisa é uma tentativa de adaptar algoritmos clássicos de otimização para resolver diretamente o problema OVO na sua formulação primal, isto é, envolvendo somente as variáveis originais. Neste sentido um primeiro passo foi alcançado, que foi adaptar o mais clássico dos algorítmos de otimização, o método de Cauchy, ou Steepest Descent. Podemos mencionar algumas aplicações que motivaram esta pesquisa:

1. Suponhamos que $\Omega$ seja um espaço de decisões e, para cada variável $x \in \Omega, f_{i}(x)$ representa uma medida de risco da decisão $x$ no cenário $i$. Suponhamos que os $m$ cenários sejam igualmente prováveis, mas mesmo que não o forem podemos reavaliar as funções adaptadas aos correspondentes cenários. A decisão minimax corresponde à escolha 
de $x$ de forma a minimizar o máximo risco possível. Esta pode ser uma alternativa muito pessimista e um trader poderia preferir descartar as piores possiblidades, de forma a proceder de forma mais realista. Por exemplo, poderíamos descartar $10 \%$ dos cenários menos prováveis, ou mais pessimistas. Isto corresponde a minimizar a função OVO de ordem $p$ com $p \approx m \times 0.10$.

2. Suponhamos que temos um problema de estimação de parâmetros onde o espaço dos parâmetros é $\Omega$ e $f_{i}(x)$ seja o erro cometido na i-ésima observação, quando adotamos o parâmetro $x$. O problema minimax de estimação corresponde a minimizar o máximo erro cometido. Como sabemos, esta estimativa é muito sensível a presença de outliers. Muitas vezes queremos eliminar, digamos, $15 \%$ dos erros maiores, pois eles podem representar observações completamente erradas. Isto corresponde a minimizar a função OVO de ordem $p$, com $p \approx 0.85 \times m$.

3. Problema de classificação de padrões. Muitas vezes alguns objetos nos são apresentados e desejamos classificá-los de acordo com determinadas categorias $1, \ldots, s$, baseados em um vetor das características $y$. Uma abordagem clássica do problema é utilizar a máxima probabilidade a posteriori: suponhamos que conhecemos a probabilidade $p(j \mid y)=$ $P$ (objeto $w$ com vetor de carateristicas $y$ ser da categoria $j$ ). Queremos atribuir ao objeto, com vetor de caracter'ssticas $y$, a categoria $j^{*}(y)=\operatorname{argmax}_{j=1, \ldots, s}\{p(j \mid y)\}$. Se as probabilidades $p(j \mid y)$ não são conhecidas, podemos estimá-las usando funções $h_{j}\left(x_{j}, y\right)$ parametrizadas pelo vetor $x_{j}$. O objetivo, então, é obter $x_{j}$ minimizando $\sum_{i=1}^{m}\left(z_{j}^{i}-h_{j}\left(x_{j}, y_{i}\right)\right)^{2}$, onde $z_{j}^{i}=1$ se $y_{i}$ é da categoria $\mathrm{j}$, e 0 caso contrário. Ou descartar algumas atribuições pouco prováveis, minimizando a função OVO de ordem $p$, por exemplo $p=95 \% m$, do conjunto de funções $\left\{f_{1}, \ldots, f_{m}\right\}$ onde $f_{i}\left(x_{j}\right)=\left(z_{j}^{i}-h_{j}\left(x_{j}, y_{i}\right)\right)^{2}$.

A tese será apresentada em 6 capítulos. No capítulo 2 formalizamos o problema OVO e apresentamos sua reformulação, denotada por OVO*, baseada na equivalência entre o problema OVO e um problema de programação matemática com restrições de equilíbrio. Analisamos a estrutura do problema reformulado e a viabilidade do uso de algoritmos já conhecidos na área de programação não linear na resolução de OVO*. No capítulo 3 derivamos condições de otimalidade de primeira e segunda ordem para o problema OVO, relacionando minimizadores de ambos os problemas. A partir desta análise sugerimos o uso de algoritmos baseados em gradiente projetado, dada a facilidade de restauração de pontos inviáveis de OVO*. 
No capítulo 4 apresentamos um teorema de equivalência entre um problema MPEC e um problema de desigualdade variacional. Definimos algumas condições nas quais asseguramos que pontos estacionários de um problema de otimização não linear (PNL) sejam soluções de um problema de desigualdade variacional (VIP) e, em particular, de OVO*. O capítulo 5 apresenta o método de Cauchy primal para resolver o problema OVO. Apresentamos o algoritmo que é, em um certo sentido, uma generalização do método do gradiente, provando sua convergência a pontos estacionários de OVO. No capítulo 6 são dados alguns exemplos numéricos de todas as abordagens sugeridas para atacar o problema, fazendo uma comparação entre estas. As conclusões e pesquisas futuras estão no capítulo 7 . 


\section{Capítulo 2}

\section{Otimização do Valor Ordenado}

\subsection{Introdução}

Começaremos com a descrição do problema de Otimização do Valor Ordenado (OVO). Suponhamos que $f_{1}, \ldots, f_{m}$ são funções contínuas a valores reais, definidas em um conjunto arbitrário $\Omega \subset \Re^{n}$. Para cada $x \in \Omega$, os valores $f_{1}(x), \ldots, f_{m}(x)$ são ordenados de forma que

$$
f_{i_{1}(x)}(x) \leq f_{i_{2}(x)}(x) \leq \ldots \leq f_{i_{m}(x)}(x) .
$$

Para qualquer valor inteiro $p \in I=\{1, \ldots, m\}$, a função OVO de ordem$p, f: \Omega \rightarrow \mathbb{R}$, é definida por

$$
f(x)=f_{i_{p}(x)}(x) .
$$

A função $f$ está bem definida, é contínua (ver Teorema 2.1.1), apesar do conjunto de índices $i_{1}, \ldots, i_{m}$ não ser único. Se $p=1, f(x)=$ $\min \left\{f_{1}(x), \ldots, f_{m}(x)\right\}$ e se $p=m$, temos $f(x)=\max \left\{f_{1}(x), \ldots, f_{m}(x)\right\}$.

Teorema 2.1.1. A função OVO de ordem-p é contínua.

Prova. Suponhamos que $x_{k} \rightarrow x$, e que, para todo $k$ em alguma subsequência,

$$
\left|f\left(x_{k}\right)-f(x)\right| \geq \delta>0 .
$$

Para esta subsequência, existe um índice $j \in\{1, \ldots, m\}$ tal que

$$
f\left(x_{k}\right)=f_{j}\left(x_{k}\right)
$$


infinitas vezes. Portanto,

$$
f_{j}\left(x_{k}\right) \geq f_{\ell}\left(x_{k}\right)
$$

para no mínimo $p$ índices $\ell \in\{1, \ldots, m\}$. Mais ainda,

$$
f_{j}\left(x_{k}\right) \leq f_{\ell}\left(x_{k}\right)
$$

para no mínimo $m-p+1$ índices $\ell$ no conjunto $\{1, \ldots, m\}$. Como o número de subconjuntos de $\{1, \ldots, m\}$ é finito, um conjunto de índices $\ell$ que verefique (2.4) é repetido infinitas vezes, e o mesmo acontece com um conjunto de índices $\ell$ que verifique (2.5). Portanto, tomando os limites em (2.4) e (2.5), concluimos que

$$
\begin{aligned}
& f_{j}(x) \geq f_{\ell}(x) \\
& \text { para, no mínimo } p \text { índices } \ell \in\{1, \ldots, m\} \text { e } \\
& \\
& f_{j}(x) \leq f_{\ell}(x)
\end{aligned}
$$

para, no mínimo, $p$ índices $\ell \in\{1, \ldots, m\}$. Portanto,

$$
f(x)=f_{j}(x) .
$$

Mas $f_{j}\left(x_{k}\right) \rightarrow f_{j}(x)$, o que contradiz (2.3).

Para enxergar melhor as características de não diferenciabilidade da função $f$, assim como a possibilidade de haver um grande número de minimizadores locais, vejamos um exemplo gráfico. Suponhamos que $m=4$, ou seja, temos quatro funções $f_{i}$, e que desejamos minimizar a função OVO de ordem 2, dada por $f(x)$. A curva em negrito da Figura 2.1 representa o gráfico de $f(x)$.

Nosso objetivo é resolver:

$$
\text { Problema OVO: Minimizar } f(x), \quad \text { s.a. } x \in \Omega \text {. }
$$

\subsection{Reformulação do problema}

Como foi visto na definição do problema OVO, pode ser bastante difícil lidar com tantos minimizadores locais, principalmente pelo fato de que muitos deles podem ser pontos onde a função OVO não é diferenciável. Nosso intuito agora é redefinir a função OVO de forma a tornar mais fácil sua otimização. A nova definição é uma consequência do seguinte lema. De agora em diante, denotemos \# como sendo o número de elementos de um conjunto. Também denotemos $e=(1, \ldots, 1)^{T} \in \mathbb{R}^{m}$. 


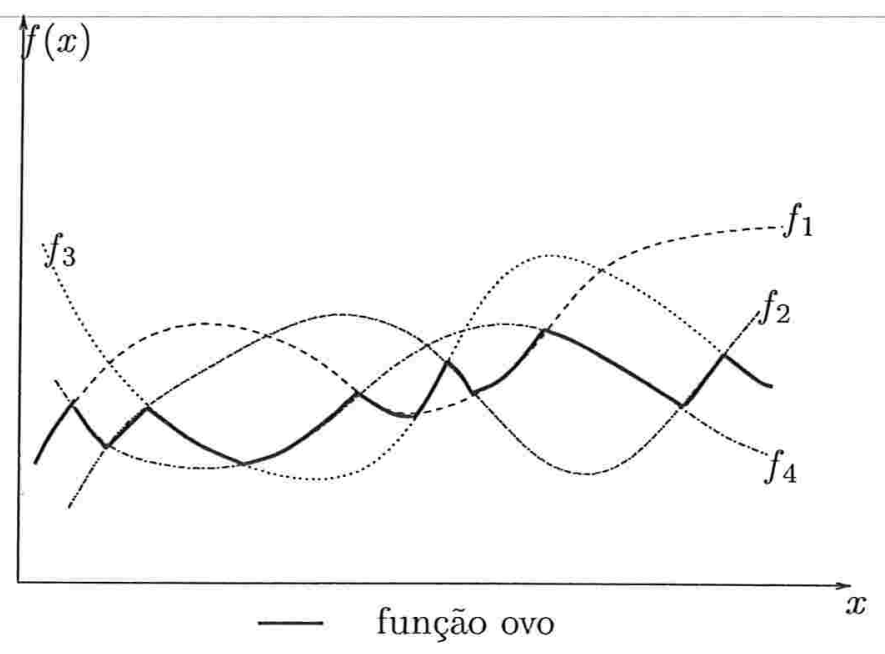

Figura 2.1: Gráfico da função OVO $f(x)$, com $m=4$ e $p=2$.

Lema 2.2.1. Suponha que $z_{1}, \ldots, z_{m}$ sejam números reais tais que

$$
z_{1} \leq z_{2} \leq \ldots \leq z_{m}
$$

Então, para qualquer $p \in\{1, \ldots, m\}$, existem vetores $r^{\prime}, u^{\prime}, w^{\prime} \in \mathbb{R}^{m}$ tais que $\left(z_{p}, r^{\prime}, u^{\prime}, w^{\prime}\right)$ é uma solução do seguinte problema:

$$
\begin{gathered}
\text { Minimizar } z \\
\text { Sujeito } a \\
\sum_{i=1}^{m} r_{i} w_{i}=0 \\
\sum_{i=1}^{m}\left(1-r_{i}\right) u_{i}=0 \\
\sum_{i=1}^{m} r_{i}=p \\
u_{i}-z+z_{i}-w_{i}=0, \quad i=1, \ldots, m \\
u \geq 0, \quad 0 \leq r \leq e \quad w \geq 0 .
\end{gathered}
$$

Prova. Defina $u^{\prime}, r^{\prime}$ e $w^{\prime}$ por 


$$
\begin{array}{ll}
r_{i}^{\prime}=1, & i=1, \ldots, p \\
r_{i}^{\prime}=0, & i=p+1, \ldots, m \\
u_{i}^{\prime}=z_{p}-z_{i}, & i=1, \ldots, p \\
u_{i}^{\prime}=0, & i=p+1, \ldots, m \\
w_{i}^{\prime}=0, & i=1, \ldots, p \\
w_{i}^{\prime}=z_{i}-z_{p}, & i=p+1, \ldots, m
\end{array}
$$

Claramente $\left(z_{p}, r^{\prime}, u^{\prime}, w^{\prime}\right)$ é um ponto viável de (2.7) para o qual a função objetivo é $z_{p}$. Agora, suponha que exista um ponto viável $(z, r, u, w)$ de (2.7) tal que $z<z_{p}$. Então, por (2.7c) e (2.7d), $(z, r, u, w)$ satisfaz

$$
w_{i}=u_{i}-z+z_{i}>0, \quad i=p, \ldots, m .
$$

Portanto, por (2.7a), temos que

$$
r_{i}=0, \quad i=p, \ldots, m .
$$

violando a equação (2.7c). Assim, $(z, r, u, w)$ não pode ser viável.

Isto significa que, para todo ponto viável $(z, r, u, w)$, temos que $z \geq z_{p}$. Desde que $\left(z_{p}, u^{\prime}, r^{\prime}, w^{\prime}\right)$ é viável, a prova está completa.

Pelo Lema 2.2.1, dado $x \in \Omega, f(x)$ é o valor de $z$ que resolve o problema (2.7). Como o problema OVO é dado por

$$
\operatorname{Minimizar}_{x \in \Omega} f(x),
$$

a reformulação proposta para resolver este problema é dada por

$$
\text { OVO }^{*}\left\{\begin{array}{l}
\text { Minimizar } z \\
\text { Sujeito a } \\
\sum_{i=1}^{m} r_{i} w_{i}=0 \\
\sum_{i=1}^{m}\left(1-r_{i}\right) u_{i}=0 \\
\sum_{i=1}^{m} r_{i}=p \\
u_{i}-z+f_{i}(x)-w_{i}=0, \quad i=1, \ldots, m \\
u \geq 0, \quad 0 \leq r \leq e \quad w \geq 0, x \in \Omega .
\end{array}\right.
$$

Chamaremos, daqui por diante, o problema reformulado de OVO*.

Observe que, em (2.7), $x$ tem valor fixo e $f(x)$ resolve o problema de minimização. Em (2.9) o minimizador é encontrado entre todos os valores possíveis de $x$. Claramente, (2.7) é um problema contínuo mas, em 
princípio, (2.9) não o é, pois $x$ pode ser tomado em um conjunto arbitrário $\Omega$.

Note que se $p=m$ então a reformulação do problema OVO é equivalente a formulação usual do problema MinMax. De fato, da terceira restrição de (2.9) e pelo fato de $0 \leq r \leq e$ temos $r=e$. Com isso a igualdade $\sum_{i=1}^{m} u_{i}(1-$ $\left.r_{i}\right)=0$ é trivialmente satisfeita. E ainda pelo fato de $\sum_{i=1}^{m} r_{i} w_{i}=0$ e de $w \geq 0, r=e$ implica que $w=0$. Assim, o problema (2.9) torna-se

$$
\begin{gathered}
\text { Minimizar } z \\
\text { s.a. }\left\{\begin{array}{l}
-z+f_{i}(x)+u_{i}=0 \quad i=1, \ldots, m \\
w \geq 0 \\
x \in \Omega
\end{array}\right.
\end{gathered}
$$

ou, equivalentemente,

$$
\begin{gathered}
\text { Minimizar } z \\
\text { s.a. }\left\{\begin{array}{l}
z \geq f_{i}(x) \quad i=1, \ldots, m \\
x \in \Omega
\end{array}\right.
\end{gathered}
$$

\subsection{Viabilidade do problema OVO*}

Nesta seção estudaremos a viabilidade do problema (2.9). A reformulação a ser usada aqui é baseada no quadrado das restrições de OVO*, mantendo a positividade das variáveis. Provaremos que pontos estacionários desta soma de quadrados são pontos viáveis para (2.9).

Para isso, considere o caso onde $\Omega \subset \mathbb{R}^{n}$ e as funções $f_{i}$ são continuamente diferenciáveis. Mais ainda, suponha que o conjunto $\Omega$ é definido por

$$
\Omega=\left\{x \in \mathbb{R}^{n} \text { tal que } h(x)=0, l_{b} \leq x \leq u_{b}\right\},
$$

onde, eventualmente, alguns limitantes possam ser infinitos e $h$ é uma função vetorial continuamente diferenciável. Praticamente todo algoritmo razoável de programação matemática para PNL tem a propriedade de que todo ponto limite é um ponto estacionário da soma dos quadrados das inviabilidades. Para o nosso problema, todo ponto limite será um ponto estacionário de primeira ordem de

$$
\text { Minimize }\left[\sum_{i=1}^{m} r_{i} w_{i}\right]^{2}+\left[\sum_{i=1}^{m}\left(1-r_{i}\right) u_{i}\right]^{2}
$$




$$
\begin{gathered}
+\left[\left(\sum_{i=1}^{m} r_{i}\right)-p\right]^{2}+\sum_{i=1}^{m}\left[u_{i}-z+f_{i}(x)-w_{i}\right]^{2}+\sum_{i=1}^{m} h_{i}^{2}(x) \\
\text { s.t. } u \geq 0, \quad 0 \leq r \leq e, \quad w \geq 0, \quad l_{b} \leq x \leq u_{b} .
\end{gathered}
$$

Portanto, é natural questionar se pontos estacionários de (2.11-2.13) representam pontos viáveis de (2.9). No próximo lema destacamos uma simples propriedade que será útil nas nossas análises.

Lema 2.3.1. Suponha que $x \in \Omega$. Então, $(z, r, u, w)$ satisfaz as condições de otimalidade de primeira ordem de

$$
\begin{gathered}
\text { Minimize }\left[\sum_{i=1}^{m} r_{i} w_{i}\right]^{2}+\left[\sum_{i=1}^{m}\left(1-r_{i}\right) u_{i}\right]^{2} \\
+\left[\left(\sum_{i=1}^{m} r_{i}\right)-p\right]^{2}+\sum_{i=1}^{m}\left[u_{i}-z+f_{i}(x)-w_{i}\right]^{2} \\
\text { s.t. } u \geq 0, \quad 0 \leq r \leq e, w \geq 0 .
\end{gathered}
$$

se e somente se $(x, z, r, u, w)$ satisfaz as condições de primeira ordem de (2.11-2.13).

Prova. Basta escrever as condições de otimalidade de (2.11-2.13) e compará-las com (2.14-2.16), considerando o fato de que $x \in \Omega$.

Devido ao Lema 2.3.1, nossa questão agora é saber se, dado $x \in \Omega$, os pontos que satisfazem as condições de otimalidade de (2.14-2.16) satisfazem as restrições de (2.9). Em outras palavras, queremos saber se com um ponto viável $x \in \Omega$ e um ponto estacionário $(z, r, u, w)$ de (2.11-2.13) podemos assegurar que $z=f_{i_{p}}(x)$. A resposta é positiva, e será estabelecida no próximo teorema.

Teorema 2.3.2. Suponha que $\Omega$ seja dado por (2.10), as funções $f_{i}$ e $h$ sejam continuamente diferenciáveis, $x \in \Omega$ e $\left(x_{*}, z_{*}, r_{*}, u_{*}, w_{*}\right)$ seja um ponto estacionário de (2.11-2.13). Então, $\left(x_{*}, z_{*}, r_{*}, u_{*}, w_{*}\right)$ satisfaz as restrições de (2.9).

Prova. Defina

$$
\theta_{1}=\sum_{i=1}^{m}\left[r_{*}\right]_{i}\left[w_{*}\right]_{i}
$$




$$
\begin{gathered}
\theta_{2}=\sum_{i=1}^{m}\left(1-\left[r_{*}\right]_{i}\right)\left[u_{*}\right]_{i} \\
\theta_{3}=\sum_{i=1}^{m}\left[r_{*}\right]_{i}-p
\end{gathered}
$$

Se $\left(x_{*}, r_{*}, u_{*}, z_{*}, w_{*}\right)$ é um ponto de Karush-Kunh-Tucker ( KKT) de (2.11-2.13), então existem $\mu_{1}, \mu_{2}, \mu_{3}, \mu_{4}$ tais que

$$
\begin{gathered}
2 \theta_{1}\left[w_{*}\right]_{i}-2 \theta_{2}\left[u_{*}\right]_{i}+2 \theta_{3}-\left[\mu_{1}\right]_{i}+\left[\mu_{2}\right]_{i}=0, \quad i=1, \ldots, m \\
2 \theta_{2}\left(1-\left[r_{*}\right]_{i}\right)+2\left(\left[u_{*}\right]_{i}-z_{*}+f_{i}\left(x_{*}\right)-\left[w_{*}\right]_{i}\right)-\left[\mu_{3}\right]_{i}=0, \quad i=1, \ldots, m \\
-2 \sum_{i=1}^{m}\left(\left[u_{*}\right]_{i}-z_{*}+f_{i}\left(x_{*}\right)-\left[w_{*}\right]_{i}\right)=0 \\
2 \theta_{1}\left[r_{*}\right]_{i}-2\left(\left[u_{*}\right]_{i}-z_{*}+f_{i}\left(x_{*}\right)-\left[w_{*}\right]_{i}\right)-\left[\mu_{4}\right]_{i}=0, \quad i=1, \ldots, m \\
{\left[\mu_{1}\right]_{i}\left[r_{*}\right]_{i}=\left[\mu_{2}\right]_{i}\left[1-\left[r_{*}\right]_{i}\right]=\left[\mu_{3}\right]_{i}\left[u_{*}\right]_{i}=\left[\mu_{4}\right]_{i}\left[w_{*}\right]_{i}=0, \quad i=1, \ldots, m} \\
u_{*} \geq 0,0 \leq r_{*} \leq e, w_{*} \geq 0, \mu_{1} \geq 0, \mu_{2} \geq 0, \mu_{3} \geq 0, \mu_{4} \geq 0
\end{gathered}
$$

(a) Suponha que $\theta_{1}=\theta_{2}=0$. Então (2.17)-(2.22) são as condições de KKT do seguinte problema convexo:

$$
\left.\operatorname{Min}\left(\left(\sum_{i=1}^{m} r_{i}\right)-p\right)\right)^{2} \text { s.a. } 0 \leq r \leq e .
$$

Como $p \in[1, m]$, temos que $\theta_{3}=0$, e a prova está completa.

(b) Suponha que $\theta_{1}=0$. Então, por (2.20) e (2.22), segue que

$$
2\left(\left[u_{*}\right]_{i}-z_{*}+f_{i}\left(x_{*}\right)-\left[w_{*}\right]_{i}\right)=-\left[\mu_{4}\right]_{i} \leq 0, \quad i=1, \ldots, m
$$

De (2.19) conclui-se que

$$
\left[u_{*}\right]_{i}-z_{*}+f_{i}\left(x_{*}\right)-\left[w_{*}\right]_{i}=0, \quad i=1, \ldots, m
$$

Multiplicando (2.18) por $\left[u_{*}\right]_{i}$ e considerando (2.21), temos

$$
2 \theta_{2}\left[u_{*}\right]_{i}\left(1-\left[r_{*}\right]_{i}\right)=0, \quad i=1, \ldots, m
$$

Portanto, pela definição de $\theta_{2}$ e por (2.22), segue que $\theta_{2}=0$. O restante da demonstração segue de maneira análoga a prova feita em (a). 
(c) Suponha que $\theta_{2}=0$. Então, por (2.19) e (2.22), segue que

$$
2\left(\left[u_{*}\right]_{i}-z_{*}+f_{i}\left(x_{*}\right)-\left[w_{*}\right]_{i}\right)=\left[\mu_{3}\right]_{i} \geq 0, \quad i=1, \ldots, m
$$

Da igualdade (2.19) conclui-se que

$$
\left[u_{*}\right]_{i}-z_{*}+f_{i}\left(x_{*}\right)-\left[w_{*}\right]_{i}=0, \quad i=1, \ldots, m
$$

Multiplicando (2.20) por $\left[w_{*}\right]_{i}$ e considerando (2.21), temos que

$$
2 \theta_{2}\left[w_{*}\right]_{i}\left[r_{*}\right]_{i}=0, \quad i=1, \ldots, m
$$

Logo, pela definição de $\theta_{1}$ e por (2.22), temos que $\theta_{1}=0$. O restante da prova segue os passos do item (a).

(d) Suponha que $\theta_{1}>0$ e $\theta_{2}>0$.

Da definição de $\theta_{1}$ segue que existe um índice $k$ tal que $\left[r_{*}\right]_{k}>0$ e $\left[w_{*}\right]_{k}>0$. Por $(2.20)$ e (2.21),

$$
2 \theta_{1}\left[r_{*}\right]_{k}=2\left(\left[u_{*}\right]_{k}-z_{*}+f_{i}\left(x_{*}\right)-\left[w_{*}\right]_{k}\right)>0
$$

$\mathrm{e}\left[\mu_{1}\right]_{k}=0$.

De (2.18) e do fato de $\theta_{2}>0$, temos que

$$
2 \theta_{2}\left(1-\left[r_{*}\right]_{k}\right)=-2\left(\left[u_{*}\right]_{k}-z_{*}+f_{k}\left(x_{*}\right)-\left[w_{*}\right]_{k}\right)+\left[\mu_{3}\right]_{k}=0 .
$$

Isto implica que $\left[\mu_{3}\right]_{k}>0$ e, considerando $(2.21)$, conclui-se que $\left[u_{*}\right]_{k}=$ 0. Assim,

$$
\left[\mu_{1}\right]_{k}=0,\left[w_{*}\right]_{k}>0 \text {, and }\left[u_{*}\right]_{k}=0 .
$$

e usando (2.17) temos

$$
2 \theta_{1}\left[w_{*}\right]_{k}+2 \theta_{3}+\left[\mu_{2}\right]_{k}=0
$$

o que implica que

$$
\theta_{3}<0
$$

Por outro lado, pela definição de $\theta_{2}$, existe $k$ tal que $1-\left[r_{*}\right]_{k}>0$ e $\left[u_{*}\right]_{k}>0$. Por $(2.18)$ e $(2.21)$,

$$
-2 \theta_{2}\left(1-\left[r_{*}\right]_{k}\right)=\left(\left[u_{*}\right]_{k}-z_{*}+f_{i}\left(x_{*}\right)-\left[w_{*}\right]_{k}\right)<0
$$

e $\left[\mu_{2}\right]_{k}=0$. Por $(2.20)$ e pelo fato de $\theta_{2}>0$, temos que

$$
2 \theta_{2}\left(1-\left[r_{*}\right]_{k}\right)=2\left(\left[u_{*}\right]_{k}-z_{*}+f_{k}\left(x_{*}\right)-\left[w_{*}\right]_{k}\right)+\left[\mu_{4}\right]_{k}
$$


Isto implica em $\left[\mu_{4}\right]_{k}>0$. Por (2.21), $\left[w_{*}\right]_{k}=0 \mathrm{e}$, assim,

$$
\left[w_{*}\right]_{k}=0 \text { e }\left[\mu_{2}\right]_{k}=0
$$

podem ser usados juntamente com (2.17) para concluir que

$$
-2 \theta_{2}\left[u_{*}\right]_{k}+2 \theta_{3}-\left[\mu_{1}\right]_{k}=0
$$

Isto implica que $\theta_{3}>0$, o que contradiz (2.23), completando a prova.

Nosso interesse é usar algoritmos de programação não linear para resolver (2.9). De acordo com o teorema 2.3.2, minimizar a soma dos quadrados das restrições, mantendo as restrições de caixa, nos garante que pontos estacionários desta soma são necessariamente pontos viáveis de OVO*. Portanto, não é difícil encontrar um método que convirja a pontos viáveis de (2.9). Mas a pergunta agora é saber a que classe pertencem estes pontos obtidos por algum algoritmo de PNL.

Suponhamos que temos um método que convirja para pontos viáveis de (2.9). Há algumas propriedades, já bastante exploradas por muitos autores, que a maioria dos algoritmos de PNL possuem. Entre elas destacamos:

Propriedade 1 Se o ponto limite é viável e os gradientes das restrições ativas são LI (linearmente independentes), então este ponto satisfaz as condições de KKT (Karush-Kuhn-Tucker) do PNL.

Propriedade 2 Se um ponto limite é viável, então ele satisfaz as condições de Fritz-John do PNL.

Os gradientes das restrições ativas de OVO* são LD (linearmente dependentes) em todos os pontos viáveis, como já havia sido observado. Portanto, um algoritmo que satisfaz a propriedade 1 não diz nada sobre convergência para OVO*. Outra característica do problema é o fato da condição Mangasarian-Fromovitz (MF) não ser satisfeita em nenhum ponto viável. Portanto, algoritmos que satisfazem a propriedade 2 não são satisfatórios.

Ou seja, no caso de problemas com restrições de equilíbrio, qualquer ponto viável pode ser ponto de acumulação de algoritmos com as propriedades acima.

Para recordar a condição de qualificação mencionada, considere o problema de programção não linear da forma 


$$
\begin{gathered}
\text { Minimizar } f(x) \\
\quad \text { Sujeito a } \\
g_{i}(x) \leq 0, \quad i=1, \ldots, m, \\
h_{j}(x)=0, \quad, j=1, \ldots, l,
\end{gathered}
$$

onde todas as funções são a valores reais e de classe $\mathcal{C}^{1}\left(\mathbb{R}^{n}\right)$. Para um ponto viável $\bar{x}$, a condição de regularidade de Mangasarian-Fromovitz é a seguinte:

(i) Existe um vetor $\bar{y} \in \mathbb{R}^{n}$ tal que

$$
\begin{gathered}
\nabla g_{i}(\bar{x})^{T} \bar{y}<0, \quad i \in I(\bar{x})=\left\{i \mid g_{i}(\bar{x})=0\right\}, \\
\nabla h_{i}(\bar{x})^{T} \bar{y}=0, \quad j=1, \ldots, l .
\end{gathered}
$$

(ii) Os gradientes $\left\{\nabla h_{j}(\bar{x})\right\}, j=1, \ldots, l$ são linearmente independentes.

No trabalho [12] de Martínez-Svaiter, uma nova condição de otimalidade que não depende de qualificação de restrições é analisada. Foi identificada em muitos algoritmos de PNL uma propriedade de convergência que diz, em poucas palavras, que o "gradiente projetado aproximado (AGP) tende a zero", projeção essa feita no conjunto tangente às restrições. Esta condição de otimalidade aliada às características do problema OVO* nos sugere o uso de algortimos que satisfaçam esta condição AGP. Também nos sugere o uso de métodos baseados em gradientes projetados, já que é fácil, a partir de um ponto $x \in \Omega$, obter um ponto viável de (2.9). Em particular, no próximo capítulo, esta última sugestão será explorada. 


\section{Capítulo 3}

\section{Condições de Otimalidade}

Neste capítulo formularemos condições de otimalidade para os problemas OVO e OVO*, explorando um pouco mais as características das restrições de (3.2). Apresentaremos condições necessárias e suficientes de otimalidade para os problemas OVO e para sua reformulação, relacionando seus respectivos minimizadores. Veremos que a partir de um ponto viável $x \in \Omega$, é possível definir um ponto $y$ viável para $\mathrm{OVO}^{*}$ através de uma restauração natural. Este fato nos permitirá pensar em algoritmos baseados em gradiente projetado, já que se torna simples a tarefa da restauração exata. Analisaremos em que circunstâncias esta projeção poderá gerar direções de descida para a função objetivo de (3.2), e assim associá-la a uma condição de otimalidade de primeira ordem. Esboçaremos um algoritmo baseado em gradiente projetado restaurado, sem no entanto provar sua convergência, mas sugerindo uma linha de futuras pesquisas no assunto.

Suponhamos que $f_{i}: \Omega \rightarrow \mathbb{R}, \Omega \in \mathbb{R}^{n}$, para todo $i=1, \ldots, m$, e relembremos que

$$
f(x)=f_{i_{p}(x)}(x)
$$

para todo $x \in \Omega$, onde

$$
f_{i_{1}(x)}(x) \leq \ldots \leq f_{i_{m}(x)}(x) .
$$

O problema OVO considerado aqui é:

$$
\text { Minimizar } f(x) \text { s.a. } x \in \Omega \text {. }
$$

e sua respectiva reformulação é o problema OVO*:

Minimizar $z$ 


$$
\text { s.a. } \begin{cases}(a) & \sum_{i=1}^{m} r_{i} w_{i}=0 \\ (b) & \sum_{i=1}^{m}\left(1-r_{i}\right) u_{i}=0 \\ (c) & \sum_{i=1}^{m} r_{i}=p \\ (d) & u_{i}-z+f_{i}(x)-w_{i}=0, \quad i=1, \ldots, m \\ (e) & u \geq 0, \quad 0 \leq r \leq e \quad w \geq 0, \quad x \in \Omega .\end{cases}
$$

\subsection{Viabilidade de OVO* a partir de OVO}

Dado $x \in \Omega$, se definirmos $z=f_{i_{p}}(x)$ torna-se simples a tarefa de encontrar valores para $u^{\prime}, r^{\prime}, w^{\prime}$ de forma a obter um ponto viável de (3.2). De fato, ordenando os valores $f_{1}(x), \ldots, f_{m}(x)$ e fazendo

$$
\begin{array}{ll}
r_{j}^{\prime}=1, & j=1, \ldots, p \\
r_{j}^{\prime}=0, & j=p+1, \ldots, m \\
u_{j}^{\prime}=f_{i_{p}}(x)-f_{i_{j}}(x), & j=1, \ldots, p \\
u_{j}^{\prime}=0, & j=p+1, \ldots, m \\
w_{j}^{\prime}=0, & j=1, \ldots, p \\
w_{j}^{\prime}=f_{i_{j}}(x)-f_{i_{p}}(x), & j=p+1, \ldots, m
\end{array}
$$

é fácil ver que o ponto $\left(x, z, r^{\prime}, u^{\prime}, w^{\prime}\right)$ é um ponto viável de (3.2).

Formalizemos este procedimento. Dado $x \in \Omega$, considere o seguinte ordenamento

$$
\begin{gathered}
f_{i_{1}(x)}(x) \leq \ldots \leq f_{i_{s}(x)}(x)<f_{i_{s+1}(x)}(x)=\ldots=f_{i_{p}(x)}(x)= \\
=\ldots=f_{i_{\ell}(x)}(x)<f_{i_{\ell+1}(x)}(x) \leq \ldots \leq f_{i_{m}(x)}(x)
\end{gathered}
$$

Quando não houver dúvidas quanto a variável $x$ em questão, abreviaremos os índices $i_{1}(x), \ldots, i_{m}(x)$ por $i_{1}, \ldots, i_{m}$, a fim de não sobrecarregar a notação.

Para facilidade de notação, defina os conjuntos

$$
\begin{gathered}
L(x)=\left\{i \in\left\{i_{1}(x), \ldots, i_{s}(x)\right\}\right\} \\
E(x)=\left\{i \in\left\{i_{s+1}(x), \ldots, i_{l}(x)\right\}\right\} \\
G(x)=\left\{i \in\left\{i_{l+1}(x), \ldots, i_{m}(x)\right\}\right\}
\end{gathered}
$$

Veremos que é natural a escolha de $\left(r^{\prime}, u^{\prime}, w^{\prime}\right)$ acima. Dado $x \in \Omega \mathrm{e}$ atribuindo o valor $z=f_{i_{p}}(x)$, de acordo com as restrições

$$
u_{i}-z+f_{i}(x)-w_{i}=0, \quad w_{i} \geq 0, \quad u_{i} \geq 0 \quad \forall i \in\{1, \ldots, m\}
$$




$$
\begin{gathered}
\sum_{i=1}^{m} u_{i}\left(1-r_{i}\right)=0, \quad r_{i} \in[0,1] \\
\sum_{i=1}^{m} w_{i} r_{i}=0
\end{gathered}
$$

concluimos o seguinte:

a. se $i \in L(x)$ então $-z+f_{i}(x)<0 \Rightarrow u_{i}>w_{i} \geq 0$. Logo $u_{i}>0$. Mas como $u_{i}\left(1-r_{i}\right)=0$, segue que $r_{i}=1$.

b. se $i \in G(x)$ então $-z+f_{i}(x)>0 \Rightarrow w_{i}>0$. Mas como $w_{i} r_{i}=0$, segue que $r_{i}=0$.

Portanto, dado um ponto $x$ viável, um vetor $y$ viável para (3.2) é dado pela seguinte

\section{RESTAURAÇÃO:}

$$
\begin{gathered}
z=f_{i_{p}}(x) \\
w_{i}=\left\{\begin{array}{l}
f_{i}(x)-f_{i_{p}}(x) \text { se } i \in G(x) \\
0 \text { se } i \in E(x) \cup L(x)
\end{array}\right. \\
u_{i}= \begin{cases}f_{i_{p}}(x)-f_{i}(x) \text { se } i \in L(x) \\
0 & \text { se } i \in E(x) \cup G(x)\end{cases} \\
r_{i}=\left\{\begin{array}{lll}
1 & \text { se } & i \in L(x) \\
0 & \text { se } & i \in G(x) \\
a_{i} \in[0,1] & \text { se } i \in E(x)
\end{array}\right.
\end{gathered}
$$

de forma que $\sum_{i=1}^{m} r_{i}=p$.

Feita uma escolha de $r$ satisfazendo (3.7), decomporemos o conjunto $E(x)$ em 3 conjuntos disjuntos

$$
\begin{gathered}
E^{0}(x)=\left\{i \in E(x) \quad \mid \quad r_{i}=0\right\} \\
E^{1}(x)=\left\{i \in E(x) \quad \mid \quad r_{i}=1\right\} \\
E^{*}(x)=\left\{i \in E(x) \quad \mid \quad r_{i} \in(0,1)\right\}
\end{gathered}
$$

onde, claramente, $E(x)=E^{0}(x) \cup E^{1}(x) \cup E^{*}(x)$. 
De acordo com esta restauração, a partir de $\bar{x}$ é imediato gerar pontos viáveis $\bar{u}$ e $\bar{w}$, mas dependendo dos valores dos índices $s, p, l$ do ordenamento (3.3) existem diversas formas de restaurar $r$. Os pontos $r$ que satisfazem a condição

$$
\sum_{i \in\left\{i_{1}, \ldots, i_{m}\right\}} r_{i}=p, \sum_{i \in\left\{i_{s+1}, \ldots, i_{\ell}\right\}} r_{i}=p-s, \quad 0 \leq r_{i} \leq 1 .
$$

geram $(\bar{x}, \bar{u}, r, \bar{w}, \bar{z})$ viável para (3.2).

Veremos que esta variável $r$ tem um importante papel na obtenção de conjuntos tangentes às restrições de 3.2 e de possíveis direções de descida para a sua função objetivo $g(y)=z$.

\subsection{Condições de otimalidade para OVO}

O objetivo desta seção é provar condições necessárias e suficientes de otimalidade para o problema OVO.

De agora em diante, suponhamos que as todas as funções $f_{i}$ são contínuas em $\Omega$.

Definição 3.2.1. Dizemos que uma sequência $\left\{x_{k}\right\}$ é viável se $\left\{x_{k}\right\} \subset \Omega$.

Definição 3.2.2. Dado $x \in \Omega$, dizemos que uma sequência viável é uma sequência de descida para a função $\phi$ em relação à $x$ se existe $k_{0} \in \mathbb{I N}$ tal que

$$
\phi\left(x_{k}\right)<\phi(x) \forall k \geq k_{0} .
$$

Denotaremos, como de costume, \#A como sendo o número de elementos do conjunto $A$.

Definição 3.2.3. Dizemos que $x^{*} \in \Omega$ é um ponto estacionário do problema OVO se, para toda sequência viável $\left\{x_{k}\right\}$ que converge para $x^{*}$,

$$
\#\left\{i \in E\left(x^{*}\right) \mid\left\{x_{k}\right\} \text { é uma sequência de descida para } f_{i}\right\}<p-\# L\left(x^{*}\right)
$$

Teorema 3.2.1. Suponhamos que $x \in \Omega$. Então, $x$ é um minimizador local do problema OVO (3.1) se, e somente se, $x$ é um ponto estacionário. 
Prova. Suponhamos que $x$ seja um minimizador local, mas que não seja um ponto estacionário. Então, existe uma sequência viável $\left\{x_{k}\right\}$ que é uma sequência de descida para todo $i \in D \subset E(x)$, onde

$$
\# D \geq p-\# L(x) \text {. }
$$

Por continuidade, existe $\varepsilon>0$ tal que

$$
f_{i}(y)<f(x) \forall i \in L(x), \quad\|y-x\| \leq \varepsilon .
$$

Mais ainda, existe $k_{0} \in \mathbb{N}$ tal que

$$
f_{i}\left(x_{k}\right)<f(x) \forall k \geq k_{0}, \quad i \in D .
$$

Se $k_{1} \geq k_{0}$ for grande o suficiente, $\left\|x_{k}-x\right\| \leq \varepsilon$, e portanto

$$
f_{i}\left(x_{k}\right)<f(x) \forall k \geq k_{1}, \quad i \in D \cup L(x) .
$$

Mas \#D $\cup L(x) \geq p$, portanto

$$
f\left(x_{k}\right)=f_{i_{p}\left(x_{k}\right)}\left(x_{k}\right)<f(x) \forall k \geq k_{1} .
$$

Isto implica que $x$ não é um minimizador local.

Agora, suponhamos que $x$ não seja um minimizador local de (3.1). Portanto, existe uma sequência viável $\left\{x_{k}\right\}$ tal que

$$
f\left(x_{k}\right)<f(x) \text { para } k \text { suficientemente grande. }
$$

Portanto, existe $k_{2} \in \mathbb{I N}$ tal que

$$
f_{i_{1}\left(x_{k}\right)}\left(x_{k}\right) \leq \ldots \leq f_{i_{p}\left(x_{k}\right)}\left(x_{k}\right)=f\left(x_{k}\right)<f(x)
$$

para todo $k \geq k_{2}$.

Pelo fato do número de diferentes conjuntos $\left\{i_{1}\left(x_{k}\right), \ldots, i_{p}\left(x_{k}\right)\right\}$ ser finito, um deles é repetido infinitas vezes em (3.9). Denotemos este conjunto por $\left\{i_{1}, \ldots, i_{p}\right\}$. Portanto, tomando uma apropriada subsequência (que é também uma sequência viável), segue que:

$$
f_{i_{1}}\left(x_{k}\right) \leq \ldots \leq f_{i_{p}}\left(x_{k}\right)=f\left(x_{k}\right)<f(x)
$$

para todo $k \geq k_{2}$.

Como $f_{i}(x)>f(x)$ para todo $i \in G(x)$, a continuidade das funções implica que o conjunto $\left\{i_{1}, \ldots, i_{p}\right\}$ não contém elementos de $G(x)$. Assim,

$$
\left\{i_{1}, \ldots, i_{p}\right\} \subset L(x) \cup E(x) .
$$


Portanto, para no mínimo $p-\# L(x)$ elementos de $E(x)$, temos que

$$
f_{i}\left(x_{k}\right)<f(x)
$$

se $k$ for suficientemente grande. Dessa forma, a sequência $x_{k}$ é de descida para no mínimo $p-\# L(x)$ funções do conjunto $E(x)$. Isto completa a prova.

Definição 3.2.4. Dizemos que $d \in \mathbb{R}^{n}$ é uma direção tangente ao conjunto $\Omega$ no ponto $x \in \Omega$ se existe uma sequência viável $\left\{x_{k}\right\}$ que converge para $x$ tal que

$$
d=\|d\| \lim _{k \rightarrow \infty} \frac{x_{k}-x}{\left\|x_{k}-x\right\|} .
$$

Os próximos teoremas estabelecem condições de otimalidade para o problema OVO usando o conceito de direções tangentes.

Teorema 3.2.2. Suponhamos que $x$ seja um minimizador local de (3.1) $e$ que $f_{i}$ tenha derivadas de primeira ordem contínuas numa vizinhança de $x$, para todo $i \in E(x)$. Então, para toda direção tangente d,

$$
\#\left\{i \in E(x) \mid\left\langle d, \nabla f_{i}(x)\right\rangle<0\right\}<p-\# L(x) .
$$

Prova. Suponhamos que a tese não seja verdadeira. Então existe uma direção tangente $d$ e um conjunto $D_{1} \subset E(x)$, tais que $\# D_{1} \geq p-\# L(x)$ e

$$
\left\langle d, \nabla f_{i}(x)\right\rangle<0
$$

para todo $i \in D_{1}$. Defina $\left\{x_{k}\right\}$ uma sequência viável que convirja para $x$ de forma que

$$
d=\|d\| \lim _{k \rightarrow \infty} \frac{x_{k}-x_{*}}{\left\|x_{k}-x_{*}\right\|}
$$

Pela diferenciabilidade de $f_{i}$, para todo $i \in D_{1}$, temos que:

$$
f_{i}\left(x_{k}\right)=f_{i}(x)+\left\langle\nabla f_{i}(x), x_{k}-x\right\rangle+o\left(\left\|x_{k}-x\right\|\right) \text {. }
$$

Portanto,

$$
\|d\| \frac{f_{i}\left(x_{k}\right)-f_{i}(x)}{\left\|x_{k}-x\right\|} \leq\left\langle\nabla f_{i}(x),\|d\| \frac{x_{k}-x}{\left\|x_{k}-x\right\|}\right\rangle+\|d\| \frac{o\left(\left\|x_{k}-x\right\|\right)}{\left\|x_{k}-x\right\|} .
$$


Tomando os limites, temos que, para $k$ suficientemente grande,

$$
\|d\| \frac{f_{i}\left(x_{k}\right)-f_{i}(x)}{\left\|x_{k}-x\right\|} \leq \frac{\left\langle\nabla f_{i}(x), d\right\rangle}{2}<0 .
$$

Logo, para $k$ suficientemente grande e para todo $i \in D_{1}$,

$$
f_{i}\left(x_{k}\right)<f_{i}(x) .
$$

Isto contradiz o Teorema 3.2.1.

Teorema 3.2.3. Suponhamos que $x$ seja um minimizador local de (3.1) $e$ que $f_{i}$ tenha derivadas contínuas de primeira e segunda ordem numa vizinhança de $x$, para todo $i \in E(x)$. Para toda direção tangente $d$, definamos

$$
D^{\prime}=\left\{i \in E(x) \mid\left\langle d, \nabla f_{i}(x)\right\rangle<0\right\}
$$

$e$

$$
D^{\prime \prime}=\left\{i \in E(x) \mid \nabla f_{i}(x)=0 \text { and } d^{T} \nabla^{2} f_{i}(x) d<0\right\}
$$

Então,

$$
\#\left(D^{\prime} \cup D^{\prime \prime}\right)<p-\# L(x) .
$$

Prova. Suponhamos que a tese não seja verdadeira. Então, existe $d \in \mathbb{R}^{n}$ uma direção tangente tal que

$$
\#\left(D^{\prime} \cup D^{\prime \prime}\right) \geq p-\# L(x) .
$$

Defina $\left\{x_{k}\right\}$ uma sequência viável que convirja para $x$ e

$$
d=\|d\| \lim _{k \rightarrow \infty} \frac{x_{k}-x}{\left\|x_{k}-x\right\|}
$$

Se $i \in D^{\prime}$, a argumentação da prova do Teorema 3.2 .2 mostra que $\left\{x_{k}\right\}$ é uma direção de descida. Suponhamos, agora, que $i \in D^{\prime \prime}$.

Pelo fato de $f_{i}$ ter derivadas de segunda ordem contínuas, segue que

$$
\begin{gathered}
f_{i}\left(x_{k}\right)=f_{i}(x)+\left\langle\nabla f_{i}(x), x_{k}-x\right\rangle+\frac{1}{2}\left(x_{k}-x\right)^{T} \nabla^{2} f(x)\left(x_{k}-x\right)+o\left(\left\|x-x_{k}\right\|^{2}\right) \\
=f_{i}(x)++\frac{1}{2}\left(x_{k}-x\right)^{T} \nabla^{2} f(x)\left(x_{k}-x\right)+o\left(\left\|x-x_{k}\right\|^{2}\right) .
\end{gathered}
$$

Logo,

$$
\frac{\left[f_{i}\left(x_{k}\right)-f_{i}(x)\right]\|d\|^{2}}{\left\|x-x_{k}\right\|^{2}}=\|d\|^{2} \frac{1}{2} \frac{\left(x_{k}-x\right)^{T}}{\left\|x-x_{k}\right\|} \nabla^{2} f(x) \frac{x_{k}-x}{\left\|x-x_{k}\right\|}+\frac{o\left(\left\|x-x_{k}\right\|^{2}\right)}{\left\|x-x_{k}\right\|^{2}} .
$$


Tomando os limites, temos que, para $k$ suficientemente grande, $f_{i}\left(x_{k}\right)<$ $f_{i}(x)$. Portanto, $\left\{x_{k}\right\}$ é uma direção de descida para $f_{i}$. Isto contradiz o Teorema 3.2.1.

Teorema 3.2.4. Suponha que $x \in \Omega$ e $f_{i}$ tenha derivadas de segunda ordem contínuas para todo $i \in E(x)$. Para todo vetor tangente $d$, defina $S(d)=$ $S_{1}(d) \cup S_{2}(d) \subset E(x)$ por

$$
S_{1}(d)=\left\{i \in E(x) \mid\left\langle d, \nabla f_{i}(x)\right\rangle>0\right\},
$$

$e$

$$
S_{2}(d)=\left\{i \in E(x) \mid \nabla f_{i}(x)=0 \quad \text { and } \quad d^{T} \nabla^{2} f_{i}(x) d>0\right\} .
$$

Suponhamos que, para todo vetor tangente $d$,

$$
\# S(d)>\# L(x)+\# E(x)-p,
$$

Então, $x$ é um minimizador local.

Prova. Suponhamos que $x$ não seja um minimizador local. Então, pelo Teorema 3.2.1, existe uma sequência de descida $\left\{x_{k}\right\}$ para, no mínimo, $p-$ $\# L(x)$ funções do conjunto $E(x)$. Definamos $S_{3} \subset E(x)$ por

$i \in S_{3}$ se e somente se $\left\{x_{k}\right\}$ é uma sequência de descida para $f_{i}$.

Então $\# S_{3} \geq p-\# L(x)$.

Tomemos uma subsequência convergente de $\left(x_{k}-x\right) /\left\|x_{k}-x\right\|$ e uma subsequência de $\left\{x_{k}\right\}$ tal que, para esta subsequência,

$$
\lim _{k \rightarrow \infty} \frac{x_{k}-x}{\left\|x_{k}-x\right\|}=d
$$

Então, $d$ é um vetor tangente. Consideremos os conjuntos $S_{1}(d)$ e $S_{2}(d)$ associados a $d$.

Se $i \in S_{1}(d)$, então $\left\langle d, \nabla f_{i}(x)\right\rangle>0$, e portanto:

$$
f_{i}\left(x_{k}\right)-f_{i}(x)=\left\langle\nabla f_{i}\left(x_{k}\right), x_{k}-x\right\rangle+o\left(\left\|x_{k}-x\right\|\right)
$$

e

$$
\frac{f_{i}\left(x_{k}\right)-f_{i}(x)}{\left\|x_{k}-x\right\|}=\left\langle\nabla f_{i}(x), \frac{x-x_{k}}{\left\|x-x_{k}\right\|}\right\rangle+\frac{o\left(\left\|x_{k}-x\right\|\right)}{\left\|x_{k}-x\right\|} .
$$


Logo, tomando os limites, observamos que $f_{i}\left(x_{k}\right)>f_{i}(x)$ para $k$ suficientemente grande. Portanto, $\left\{x_{k}\right\}$ não é uma sequência de descida.

Analogamente, se $i \in S_{2}(d)$,

$$
\begin{aligned}
f_{i}\left(x_{k}\right)-f_{i}(x) & =\left\langle\nabla f_{i}(x), x_{k}-x\right\rangle+\frac{1}{2}\left(x_{k}-x\right)^{T} \nabla^{2} f_{i}(x)\left(x_{k}-x\right)+o\left(\left\|x_{k}-x\right\|^{2}\right) \\
& =\frac{1}{2}\left(x_{k}-x\right)^{T} \nabla^{2} f_{i}(x)\left(x_{k}-x\right)+o\left(\left\|x_{k}-x\right\|^{2}\right) .
\end{aligned}
$$

Dividindo por $\left\|x_{k}-x\right\|^{2}$, pegando uma subsequência convergente de ( $x_{k}-$ $x) /\left\|x_{k}-x\right\|$ e tomando os limites, concluimos que $f_{i}\left(x_{k}\right)>f_{i}(x)$ para $k$ suficientemente grande. Assim, $\left\{x_{k}\right\}$ não é uma sequência de descida.

Logo, $\left(S_{1} \cup S_{2}\right) \cap S_{3}=\emptyset$, o que é impossível visto que $\# E(x) \geq$ $\# S(d)+\# S_{3}>\# L(x)+\# E(x)-p+p-\# L(x)=\# E(x)$ é uma contradição.

\subsection{Condição de otimalidade para OVO*}

Agora uma questão interessante é saber qual é a relação entre minimizadores locais do problema OVO original (3.1) e minimizadores locais do problema reformulado (3.2). Os próximos teoremas nos dizem um pouco a respeito dessa relação.

\subsubsection{Relacionando minimizadores de OVO e OVO*}

Suponha que $x^{*}$ seja um ponto viável de OVO. Defina o seguinte conjunto:

$$
S\left(x^{*}\right)=\left\{\left(z, x^{*}, r, u, w\right) \text { viável para OVO* tal que } z=f\left(x^{*}\right)\right\}
$$

onde $f(x) \stackrel{\text { def }}{=} f_{i_{p}}(x)$ para todo $x \in \Omega$.

Teorema 3.3.1. O ponto $x^{*} \in \Omega$ é um minimizador local do problema 3.1 se e somente se todos os pontos do conjunto $S\left(x^{*}\right)$ são minimizadores locais do problema reformulado 3.2.

Prova. Suponha que exista algum ponto $y \in S\left(x^{*}\right)$ que não seja mínimo local da reformulação (3.2), onde definimos $y=\left(z^{*}, x^{*}, \bar{r}, \bar{u}, \bar{w}\right)$. Então existe uma sequência de pontos viáveis $\left\{\left(z^{k}, x^{k}, r^{k}, u^{k}, w^{k}\right)\right\}_{k \in \mathbb{N}}$ tal que 


$$
\left(z^{k}, x^{k}, r^{k}, u^{k}, w^{k}\right) \longrightarrow\left(z^{*}, x^{*}, \bar{r}, \bar{u}, \bar{w}\right) \text { e que } z^{k}<z^{*} \forall k \in \mathbb{I N} .
$$

Defina o conjunto $I\left(x^{k}\right)=\left\{i \mid f_{i}\left(x^{k}\right) \leq z^{k}\right\}$.

Se temos $r_{i}^{k}>0$ então, da igualdade (a) em (3.2) segue que $w_{i}^{k}=0$. Já da equação $(d)$ segue que

$$
u_{i}^{k}-z^{k}+f_{i}\left(x^{k}\right)=0 \Rightarrow f_{i}\left(x^{k}\right) \leq z^{k}
$$

Assim, das restrições (c) e (e) de 3.2 temos que

$$
\# I\left(x^{k}\right) \geq p
$$

o que implica em $f\left(x^{k}\right) \leq z^{k}$. Portanto $x^{*}$ não é um minimizador local de OVO, contradizendo a hipótese.

Reciprocamente, suponha que $x^{*}$ não seja um minimizador local do problema OVO. Então $\exists\left\{x_{k}\right\}_{k \in \mathbb{N}} \subset \Omega$ tal que $x^{k} \longrightarrow x^{*}$ e $f\left(x^{k}\right)<f\left(x^{*}\right)$ para todo $k$.

Defina o conjunto $I\left(x^{k}\right)=\left\{i \mid f_{i}\left(x^{k}\right) \leq f\left(x^{k}\right)\right\}$. É fácil ver que \#I( $\left.x^{k}\right) \geq$ $p$, pois a sequência $x^{k}$, sendo infinita, nos possibilita escolher um conjunto de índices $J$ de forma que $\# J=p$ e tomar uma subsequência $x^{k}, k \in K_{1} \subset \mathbb{N}$, tal que $J \subset I\left(x^{k}\right)$ para todo $k \in K_{1}$. Desta forma, definimos a seguinte sequência $\left\{y^{k}=\left(z^{k}, x^{k}, r^{k}, u^{k}, w^{k}\right)\right\}$ para $k \in K_{1}$ :

$$
\begin{array}{rll}
r_{i}^{k}=1 \quad \text { se } & i \in J \\
r_{i}^{k}=0 \quad \text { se } & i \notin J \\
u_{i}^{k}=f\left(x^{k}\right)-f_{i}\left(x^{k}\right) & \text { se } \quad i \in J \\
u_{i}^{k}=0 \quad \text { se } & i \notin J \\
w_{i}^{k}=0 \quad \text { se } & i \in J \\
w_{i}^{k}=f_{i}\left(x^{k}\right)-f\left(x^{k}\right) & \text { se } \quad i \notin J
\end{array}
$$

Estes pontos $y^{k}$ definidos acima são viáveis para o problema 3.2. Pela continuidade da função OVO, estes pontos convergem para:

$$
\begin{gathered}
x^{k} \longrightarrow x^{*}, \quad z^{k} \longrightarrow z^{*}=f\left(x^{*}\right) \\
r_{i}^{k} \longrightarrow \bar{r}_{i}= \begin{cases}1 & \text { se } i \in J \\
0 & \text { se } i \notin J\end{cases}
\end{gathered}
$$




$$
\begin{aligned}
u_{i}^{k} \longrightarrow \bar{u}_{i} & = \begin{cases}f\left(x^{k}\right)-f_{i}\left(x^{k}\right) & \text { se } i \in J \\
0 & \text { se } i \in J\end{cases} \\
w_{i}^{k} \longrightarrow \bar{w}_{i} & = \begin{cases}0 & \text { se } i \notin J \\
f_{i}\left(x^{k}\right)-f\left(x^{k}\right) & \text { se } i \notin J\end{cases}
\end{aligned}
$$

Portanto, $y=\left(z^{*}, x^{*}, \bar{r}, \bar{u}, \bar{w}\right) \in S\left(x^{*}\right)$, onde $z^{*}=f\left(x^{*}\right)$. Mas $z^{k}<z^{*}$, $\forall k \in K_{1}$. Logo $y$ não é um minimizador local, o que contradiz a hipótese.

Veja que o fato de existir $y=(z, x, r, u, w) \in \Gamma$ ponto estacionário de (3.2) não implica que $x$ seja um minimizador local de (3.1). Veja um exemplo deste fato.

EXEMPLO: Considere as seguintes funções

$$
f_{1}(x)=x, \quad f_{2}(x)=2 x, \quad f_{3}(x)=3 x
$$

no intervalo $x \in[-10,10]$. Neste caso, o problema OVO* usando $p=2$ é o seguinte:

$$
\begin{aligned}
& \text { Minimizar } z \\
& \text { (1) } \quad r_{1} w_{1}+r_{2} w_{2}+r_{3} w_{3}=0 \\
& \text { (2) } \quad\left(1-r_{1}\right) u_{1}+\left(1-r_{2}\right) u_{2}+\left(1-r_{3}\right) u_{3}=0 \\
& \text { (3) } \quad r_{1}+r_{2}+r_{3}=2 \\
& \text { (4) } u_{1}-z+x-w_{1}=0 \\
& \text { s.a. (5) } u_{2}-z+2 x-w_{2}=0 \\
& \text { (6) } \quad u_{3}-z+3 x-w_{3}=0 \\
& \text { (7) } \quad u_{i} \geq 0, \quad 0 \leq r_{i} \leq e \quad w_{i} \geq 0, \quad i=1,2,3 \\
& \text { (8) } x \in[-10,10]
\end{aligned}
$$

Considere o ponto $\bar{y}=(\bar{x}, \bar{z}, \bar{r}, \bar{u}, \bar{w})=(0,0,(1,0,1),(0,0,0),(0,0,0))$, onde claramente $\bar{x}$ não é um minimizador local de OVO. Porém, provaremos que $\bar{y}$ é um minimizador local de (3.11).

Considere a sequência $y^{k}=\left(x_{k}, z_{k}, r_{k}, u_{k}, w_{k}\right) \longrightarrow \bar{y}$. Dado que $\bar{r}_{1}=$ $\bar{r}_{3}=1$, segue que $\exists k_{0} \in \mathbb{N}$ tal que

$$
r_{1}^{k}+r_{3}^{k} \geq 1.5, \quad r_{1}^{k}>0 \text { e } r_{3}^{k}>0 \forall k \geq k_{0}
$$

Da equação (1), temos que

$$
w_{1}^{k}=w_{3}^{k}=0, \quad \forall k \geq k_{0}
$$


De (3) e do fato de $r_{1}^{k}+r_{3}^{k} \geq 1.5$, seque que $r_{2}^{k}<1, \forall k \geq k_{0}$. Considerando (2) temos que $u_{2}^{k}=0, \forall k \geq k_{0}$. Com estas informações e considerando as equações (4), (5) e (6) concluimos que

$$
\begin{aligned}
& \text { (9) } u_{1}^{k}-z^{k}+x^{k}=0 \\
& \text { (10) }-z^{k}+2 x^{k}-w_{2}^{k}=0 \\
& \text { (11) } u_{3}^{k}-z^{k}+3 x^{k}=0
\end{aligned}
$$

e, portanto,

$$
\begin{array}{ll}
\text { (12) } & x^{k}=-u_{1}^{k}+z^{k} \\
\text { (13) } & z^{k}=2 x^{k}-w_{2}^{k} \\
\text { (14) } & x^{k}=\frac{1}{3}\left(u_{3}^{k}-z^{k}\right)
\end{array}
$$

Substituindo (12) em (13) e (14) em (11), seque que

$$
\begin{gathered}
z^{k}=2 z^{k}-2 u_{1}^{k}-w_{2}^{k} \Rightarrow z^{k}=2 u_{1}^{k}+w_{2}^{k} \mathrm{e} \\
z^{k}=\frac{2}{3} z^{k}-\frac{2}{3} u_{3}^{k}-w_{2}^{k} \Rightarrow z^{k}=-2 u_{3}^{k}-3 w_{2}^{k} \\
\Rightarrow z^{k}=0 \quad \forall k \geq k_{0} .
\end{gathered}
$$

o que termina a prova.

Analisemos agora o caso específico de haver uma única função $f_{i}$ tal que $f_{i}\left(x^{*}\right)=f\left(x^{*}\right)$. Vejamos que neste caso o conjunto $S\left(x^{*}\right)$ é formado por um único ponto.

Lema 3.3.2. Considere o ponto $x^{*} \in \Omega$ tal que $\# E\left(x^{*}\right)=1$, ou seja,

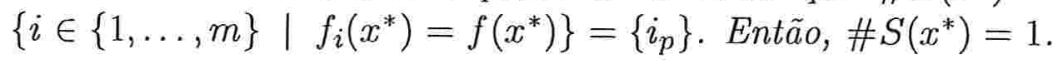

Prova. Seja $z^{*}=f\left(x^{*}\right)$ e considere o conjunto

$$
\mathcal{I}=L\left(x^{*}\right) \cup E\left(x^{*}\right)=\left\{i \mid f_{i}\left(x^{*}\right) \leq f\left(x^{*}\right)\right\}
$$

Como $\# \mathcal{I}=p$, segue que

$$
\begin{gathered}
u_{i}^{*}-z^{*}+f_{i}\left(x^{*}\right)-w_{i}^{*}=0 \Rightarrow u_{i}^{*}>0, \quad \forall i \in \mathcal{I} \backslash\left\{i_{p}\right\} \\
\Rightarrow r_{i}^{*}=1, \quad \forall i \in \mathcal{I} \backslash\left\{i_{p}\right\}
\end{gathered}
$$

Por outro lado temos

$$
\begin{gathered}
u_{i}^{*}-z^{*}+f_{i}\left(x^{*}\right)-w_{i}^{*}=0 \Rightarrow w_{i}^{*}>0, \quad \forall i \in\{1, \ldots, m\} \backslash \mathcal{I} \\
\Rightarrow r_{i}^{*}=0, \quad \forall i \in\{1, \ldots, m\} \backslash \mathcal{I} \Rightarrow r_{i_{p}}^{*}=0
\end{gathered}
$$


Portanto, a única escolha para $r$ é dada por

$$
r_{i}^{*}= \begin{cases}1 & \text { se } i \in \mathcal{I} \\ 0 & \text { se } i \in\{1, \ldots, m\} \backslash \mathcal{I}\end{cases}
$$

Consequentemente, pela viabilidade de $y^{*}$ para OVO*,

$$
u_{i}^{*}= \begin{cases}z^{*}-f_{i}\left(x^{*}\right) & \text { se } i \in \mathcal{I} \\ 0 & \text { se } i \in\{1, \ldots, m\} \backslash \mathcal{I}\end{cases}
$$

$\mathrm{e}$

$$
w_{i}^{*}= \begin{cases}0 & \text { se } i \in \mathcal{I} \\ f_{i}\left(x^{*}\right)-f\left(x^{*}\right) & \text { se } i \in\{1, \ldots, m\} \backslash \mathcal{I}\end{cases}
$$

Portanto, $y^{*}=\left(z^{*}, x^{*}, r^{*}, u^{*}, w^{*}\right)$ é o único ponto do conjunto $S\left(x^{*}\right)$.

Derivaremos agora uma condição de otimalidade de segunda ordem, baseado no comportamento das hessianas das funções pertencentes ao conjunto de empates $E(x)$. Para prová-la, entretanto, precisaremos do seguinte teorema de alternativa (ver [11]).

Teorema 3.3.3 (Gale). Dada uma matriz $l \times n$ e dado um vetor $c \in \mathbb{R}^{l}$, uma e apenas uma das seguintes proposiçôes é verdadeira:

I. Ax $\leq$ c tem um solução $x \in \mathbb{R}^{n}$.

II. $A^{T} y=0, c y=-1, y \geq 0$ tem uma solução $y \in \mathbb{R}^{l}$.

Teorema 3.3.4. Suponha que o ponto $x^{*} \in \Omega=\mathbb{R}^{n}$ seja um ponto estacionário de (3.1). Então, um ponto da forma $y^{*}=\left(z^{*}, x^{*}, r, u, w\right)$ com $z^{*}=f\left(x^{*}\right)$, viável para o problema (3.2), satisfaz as condições de KKT de (3.2).

Prova. Comecemos reescrevendo o problema 3.2 a fim de facilitar referência:

Minimizar $z$

Sujeito a 


$$
\begin{gathered}
\sum_{i=1}^{m} r_{i} w_{i}=0 \\
\sum_{i=1}^{m}\left(1-r_{i}\right) u_{i}=0 \\
\sum_{i=1}^{m} r_{i}=p \\
u_{i}-z+f_{i}(x)-w_{i}=0, \quad i=1, \ldots, m \\
u \geq 0, \quad 0 \leq r \leq e \quad w \geq 0 .
\end{gathered}
$$

Devemos provar que existem multiplicadores $\gamma, \beta, \rho$ reais, associados às restrições (3.12a), (3.12b) e (3.12c), respectivamente, vetor $\lambda \in \mathbb{R}^{m}$, associado às restrições $(3.12 \mathrm{~d})$, e $\theta, \pi, \mu^{1}, \mu^{2} \in \mathbb{R}_{+}^{m}$ referentes à restrições de caixa e sinal (3.12e), de forma que satisfaçam as seguintes equações:

$$
\begin{array}{ll}
\text { (1) } & \sum_{i=1}^{m} \lambda_{i}=1 \\
(2) & \sum_{i=1}^{m} \lambda_{i} \nabla f\left(x^{*}\right)=0 \\
(3) & w_{i} \gamma-u_{i} \beta+\rho-\mu_{i}^{1}+\mu_{i}^{2}=0 \\
(4) & \left(1-r_{i}\right) \beta+\lambda_{i}-\theta_{i}=0 \\
(5) & r_{i} \gamma-\lambda_{i}-\pi_{i}=0 \\
(6) & u_{i} \theta_{i}=w_{i} \pi_{i}=r_{i} \mu_{i}^{1}=\left(1-r_{i}\right) \mu_{i}^{2}=0 \\
& \theta \geq 0, \pi \geq 0, \mu^{1} \geq 0, \mu^{2} \geq 0
\end{array}
$$

A viabilidade de $y^{*}$ implica em específicos valores para suas componentes $r, u$ e $w$ nos conjuntos $L\left(x^{*}\right), E^{*}\left(x^{*}\right), E^{0}\left(x^{*}\right), E^{1}\left(x^{*}\right)$ e $G\left(x^{*}\right)$ da seguinte forma:

- Se $i \in L\left(x^{*}\right)$, então, por (3.12d),

$$
u_{i}=w_{i}+z^{*}-f_{i}\left(x^{*}\right)=u_{i}+f\left(x^{*}\right)-f_{i}\left(x^{*}\right)>0, \quad \text { pois } w_{i} \geq 0 .
$$

Por $(3.12 \mathrm{~b})$ temos que $u_{i}\left(1-r_{i}\right)=0$, e portanto, considerando que $u_{i}>0$, temos que $r_{i}=1$. Então, por (3.12a) temos que

$$
w_{i} r_{i}=0 \Rightarrow w_{i}=0 \text {. }
$$

- Se $i \in G\left(x^{*}\right)$, então, por (3.12d),

$$
w_{i}=u_{i}-z^{*}+f_{i}\left(x^{*}\right)=u_{i}-f\left(x^{*}\right)+f_{i}\left(x^{*}\right)>0
$$

Por (3.12a) temos que $r_{i} w_{i}=0$, e portanto, considerando que $w_{i}>0$, temos que $r_{i}=0$. Então, por (3.12b) temos que

$$
u_{i}\left(1-r_{i}\right)=0 \Rightarrow u_{i}=0 .
$$


- Se $i \in E\left(x^{*}\right)$, então, por (3.12d),

$$
w_{i}=u_{i}-z^{*}+f_{i}\left(x^{*}\right)=u_{i}
$$

Agora, suponha que $w_{i}>0$. Por (3.12a) temos que $r_{i} w_{i}=0$, e portanto, $r_{i}=0$. Assim, por (3.12b) segue que $u_{i}=0$, o que contradiz o fato de $w_{i}=u_{i}$. Portanto, $u_{i}=w_{i}=0$.

Um resumo dos valores das componentes de $y^{*}$ estão na Tabela 3.1.

\begin{tabular}{|c|c|c|c|c|c|}
\hline & $i \in L\left(x^{*}\right)$ & $i \in E^{*}\left(x^{*}\right)$ & $i \in E^{1}\left(x^{*}\right)$ & $i \in E^{0}\left(x^{*}\right)$ & $i \in G\left(x^{*}\right)$ \\
\hline$r_{i}$ & 1 & 1 & $(0,1)$ & 0 & 0 \\
\hline$u_{i}$ & $(0, \infty)$ & 0 & 0 & 0 & 0 \\
\hline$w_{i}$ & 0 & 0 & 0 & 0 & $(0, \infty)$ \\
\hline
\end{tabular}

Tabela 3.1: Possíveis valores das variáveis.

Seja $\lambda_{i}^{*}, i \in E^{1}\left(x^{*}\right) \cup E^{*}\left(x^{*}\right)$ solução do seguinte sistema:

$$
\lambda_{i}^{*} \geq 0, \quad \sum_{i \in i \in E^{1}\left(x^{*}\right) \cup E^{*}\left(x^{*}\right)} \lambda_{i}^{*}=1, \quad \sum_{i \in i \in E^{1}\left(x^{*}\right) \cup E^{*}\left(x^{*}\right)} \lambda_{i}^{*} \nabla f_{i}\left(x^{*}\right)=0
$$

Tal solução $\lambda^{*}$ existe, pois, caso contrário, pelo Teorema 3.3.3, existiria um vetor $d$ tal que

$$
\nabla f_{i}\left(x^{*}\right)^{T} d \leq-1<0, \quad \forall i \in E^{*}\left(x^{*}\right) \cup E^{1}\left(x^{*}\right)
$$

Assim sendo, de acordo com o teorema 3.2.1, essa afirmação contradiria a hipótese de $x^{*}$ ser ponto estacionário de OVO.

Agora, a partir destes valores de $\lambda^{*}$, definiremos os multiplicadores

$$
\begin{gathered}
\lambda_{i}= \begin{cases}0 & \text { se } i \in L\left(x^{*}\right) \cup G\left(x^{*}\right) \cup E_{0}\left(x^{*}\right) \\
\lambda_{i}^{*} & \text { se } i \in E^{1}\left(x^{*}\right) \cup E^{*}\left(x^{*}\right)\end{cases} \\
\gamma=\max _{i \in E^{1}\left(x^{*}\right) \cup E^{*}\left(x^{*}\right)} \frac{\lambda_{i}}{r_{i}} \\
\beta=0 \\
\rho=0
\end{gathered}
$$

e os demais de acordo com a Tabela 3.2.

Vejamos que com estas definições podemos garantir a existência de um ponto KKT. 


\begin{tabular}{|c|c|c|c|c|c|}
\hline & $i \in L\left(x^{*}\right)$ & $i \in E^{1}\left(x^{*}\right)$ & $i \in E^{*}\left(x^{*}\right)$ & $i \in E^{0}\left(x^{*}\right)$ & $i \in G\left(x^{*}\right)$ \\
\hline$\mu_{i}^{1}$ & 0 & 0 & 0 & 0 & $w_{i} \gamma \geq 0$ \\
\hline$\mu_{i}^{2}$ & 0 & 0 & 0 & 0 & 0 \\
\hline$\theta$ & 0 & $\lambda_{i} \geq 0$ & $\lambda_{i} \geq 0$ & 0 & 0 \\
\hline$\pi$ & $\gamma$ & $\gamma-\lambda_{i} \geq 0$ & $r_{i} \gamma-\lambda_{i} \geq 0$ & 0 & 0 \\
\hline
\end{tabular}

Tabela 3.2: Valores dos multiplicadores.

Por (3.13) e (3.14) segue que as equações (1) e (2) são satisfeitas.

Pelas Tabelas 3.2 e 3.1 as equações de complementaridade (6) se cumprem, para todo $i \in L\left(x^{*}\right) \cup E^{1}\left(x^{*}\right) \cup E^{*}\left(x^{*}\right) \cup E^{0}\left(x^{*}\right)$.

Analisemos as outras equações.

(i) Equação (3):

- $i \in L\left(x^{*}\right)$

$$
w_{i} \gamma-u_{i} \beta+\rho-\mu_{i}^{1}+\mu_{i}^{2}=0 \gamma-u_{i}(0)+0-0+0=0
$$

- $i \in E\left(x^{*}\right)$

$$
w_{i} \gamma-u_{i} \beta+\rho-\mu_{i}^{1}+\mu_{i}^{2}=(0) \gamma-0+0-0+0=0
$$

- $i \in G\left(x^{*}\right)$

$$
w_{i} \gamma-u_{i} \beta+\rho-\mu_{i}^{1}+\mu_{i}^{2}=w_{i}(0)-0+0-0+0=0
$$

(ii) Equação (4):

- $i \in L\left(x^{*}\right)$

$$
\left(1-r_{i}\right) \beta+\lambda_{i}-\theta_{i}=(0)(0)+(0)-(0)=0
$$

- $i \in E^{1}\left(x^{*}\right) \cup E^{*}\left(x^{*}\right)$

$$
\left(1-r_{i}\right) \beta+\lambda_{i}-\theta_{i}=\left(1-r_{i}\right)(0)+\lambda_{i}-\lambda_{i}=0
$$

- $i \in E^{0}\left(x^{*}\right)$

$$
\left(1-r_{i}\right) \beta+\lambda_{i}-\theta_{i}=(1-0)(0)+\lambda_{i}-\lambda_{i}=0
$$


- $i \in G\left(x^{*}\right)$

$$
\left(1-r_{i}\right) \beta+\lambda_{i}-\theta_{i}=(1-0)(0)+0-0=0
$$

(iii) Equação (5):

- $i \in L\left(x^{*}\right)$

$$
r_{i} \gamma-\lambda_{i}-\pi_{i}=\gamma-0-\gamma=0
$$

- $i \in E^{1}\left(x^{*}\right) \cup E^{*}\left(x^{*}\right)$

$$
r_{i} \gamma-\lambda_{i}-\pi_{i}=r_{i} \gamma-\lambda_{i}-\left(r_{i} \gamma-\lambda_{i}\right)=0
$$

- $i \in E^{0}\left(x^{*}\right) \cup G\left(x^{*}\right)$

$$
r_{i} \gamma-\lambda_{i}-\pi_{i}=(0) \gamma-0-0=0
$$

Com isso terminamos a prova.

\subsubsection{Particularidades do caso convexo}

Uma questão interessante é considerar o caso onde todas as funções $f_{i}$, $i \in I=\{1, \ldots, m\}$, são convexas e $\Omega$ também é um conjunto convexo. Nestas condições gostaríamos de saber se a função OVO de ordem $p$ tem minimizadores locais, para algum valor de $p \in I$. Para esta pergunta temos o seguinte resultado.

Teorema 3.3.5. Suponha que todas as funções $f_{i}, i \in I$, sejam convexas, $e$ todas possuem um minimizador global comum, o ponto $x^{*} \in \Omega$. Então todo minimizador local estrito da função OVO de ordem-p é também um minimizador global, para todo $p \in I$.

Prova. Defina a função

$$
\phi(\lambda)=f\left(x^{*}+\lambda d\right), \quad \lambda \in \mathbb{R}, \quad \lambda \geq 0
$$

onde $d$ é uma direção arbitrária. Provemos que $\phi(\lambda)$ é não-descrescente. Com efeito, suponha que a conjectura não seja verdadeira, ou seja, $\phi($.$) é$ crescente. Então existe um ponto $\mu$ tal que, para $t \geq 0$ suficientemente pequeno,

$$
\phi(\mu+t)<\phi(\mu)
$$


Considere $\delta>0$ suficientemente pequeno para garantir que as funções que definem $f\left(x^{*}\right)$ sejam as únicas funções que podem definir $f(x)$ na vizinhança $\delta$ de $x(\lambda)=x^{*}+\lambda d$, ou seja, $\left\{i \mid f_{i}(x)=f(x), x \in B_{\delta}\left(x^{*}\right)\right\} \subset$ $\left\{i \mid f_{i}\left(x^{*}\right)=f\left(x^{*}\right)\right\}$. Com esta vizinhança, pela continuidade e convexidade de $\phi$, segue que $\phi(\mu+t) \geq \phi(\mu)$. Portanto, a hipótese é absurda.

Como $\phi$ é não decrescente em todas as direções $d$, em particular ela é não decrescente na direção definida por um eventual minimizador local estrito diferente de $x^{*}$. Isto contradiz a definição de minimizador local estrito.

Observe que se considerarmos minimizadores não estritos no teorema 3.3.5, a conjectura não é verdadeira. Isto é facilmente verificado se considerarmos $f_{1}(x)=x^{2}, f_{2}(x)=1$ e $p=1$. Todos os pontos tais que $|x|>1$ são minimizadores locais não-globais de $f(x)$.

A seguir, um exemplo geométrico do resultado do teorema 3.3.5. Considere o problema de minimizar a função OVO de ordem $2 \mathrm{em} \mathbb{R}^{2}$, dados que as funções envolvidas são:

$$
\begin{gathered}
f_{1}\left(x_{1}, x_{2}\right)=\left(3 x_{1}+4 x_{2}-7\right)^{2} \\
f_{2}\left(x_{1}, x_{2}\right)=\left(2 x_{1}-x_{2}-1\right)^{2} \\
f_{3}\left(x_{1}, x_{2}\right)=\left(-2 x_{1}-5 x_{2}+7\right)^{2}
\end{gathered}
$$

Observe que todas as funções possuem o mesmo minimizador global, o ponto $x^{*}=(1,1)$. Na Figura 3.1 temos as curvas de nível da função OVO de ordem 2.

A solução do problema é o mínimo global $x^{*}=(1,1)$.

\subsection{Restauração e conjunto tangente}

A facilidade de restauração de um vetor $y$ a partir de sua componente $x$, como visto na seção 3.1, nos motiva a usar algoritmos baseados em gradiente projetado no conjunto tangente à (3.2), já que se torna fácil restaurar um ponto neste conjunto. Veremos que existe uma relação direta entre esta análise e as condição de otimalidade de primeira ordem, dada na seção 3.2. Mais ainda, é fácil caracterizar o conjunto tangente às restrições de (3.2) em um ponto $y$ obtido a partir de uma restauração.

Para começar, defina $C(y)=C(x, z, r, u, w)$ como sendo: 


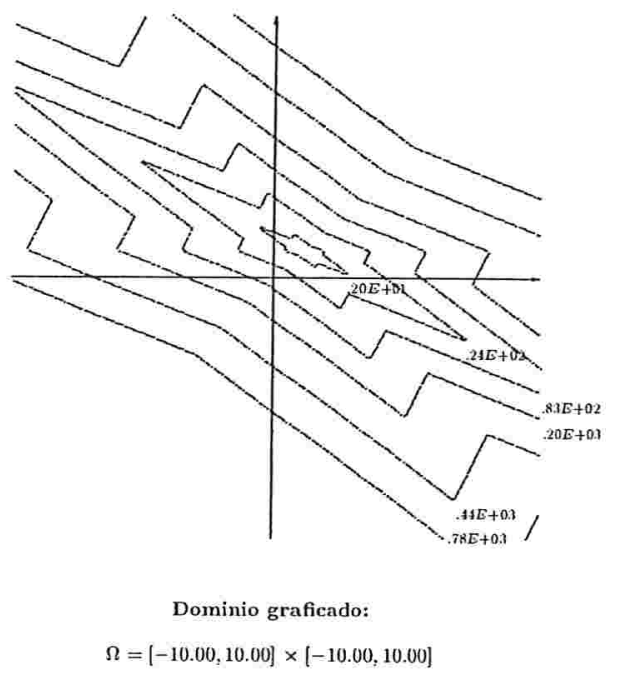

Figura 3.1: Curvas de Nível da funcao OVO, onde $\mathrm{m}=3$ e $\mathrm{p}=2$.

$$
C(y)=\left[\begin{array}{c}
\sum_{i=1}^{m} r_{i} w_{i} \\
\sum_{i=1}^{m}\left(1-r_{i}\right) u_{i} \\
\sum_{i=1}^{m} r_{i}-p \\
u_{1}-z+f_{1}(x)-w_{1} \\
\vdots \\
u_{m}-z+f_{m}(x)-w_{m}
\end{array}\right]
$$


e fixe um ponto viável restaurado $\bar{y}=(\bar{x}, \bar{z}, \bar{r}, \bar{u}, \bar{w})^{T}$.

Queremos caracterizar o plano $\Pi$ definido por

$$
\Pi(\bar{y})=\left\{y \in \Gamma \mid C^{\prime}(\bar{y})(y-\bar{y})=0\right\}
$$

onde $C^{\prime}$ é o Jacobiano de $C(y)$ e $\Gamma=\left\{(x, z, r, u, w) \in \Re^{n+3 m+1} \mid x \in \Omega, 0 \leq\right.$ $r \leq e, u \geq 0, w \geq 0\}$.

Lema 3.4.1. O conjunto tangente às restrições de (3.2) em um ponto $\bar{y}$ viável obtido por (3.4-3.7) é dado pela seguinte região poliedral:

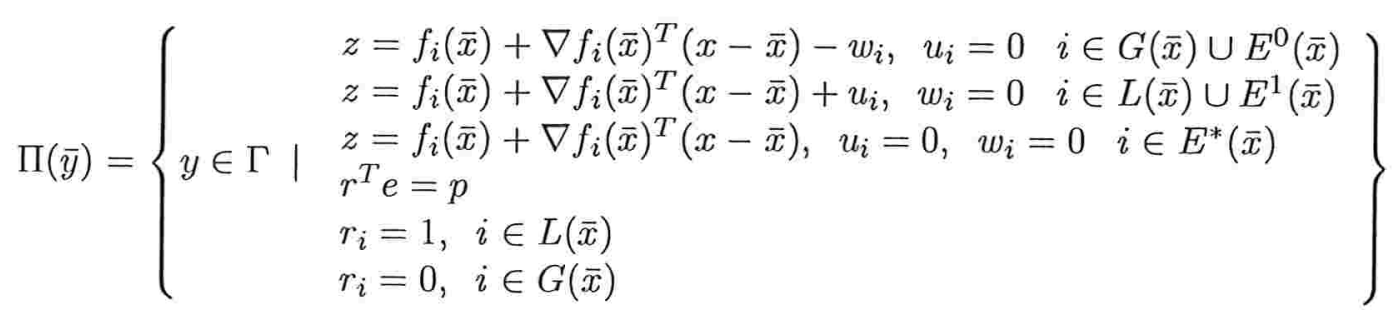

Prova:

As equações do plano tangente $C^{\prime}(\bar{y})(y-\bar{y})=0$ no ponto $\bar{y}$ são:

$$
\begin{array}{r}
\bar{w}^{T}(r-\bar{r})+\bar{r}^{T}(w-\bar{w})=0 \\
-\bar{u}^{T}(r-\bar{r})+(e-\bar{r})^{T}(u-\bar{u})=0 \\
e^{T}(r-\bar{r})=0 \\
\nabla f_{i}(\bar{x})^{T}(x-\bar{x})-z+\bar{z}+u_{i}-\bar{u}_{i}-w_{i}+\bar{w}_{i}, \quad i=1, \ldots, m \\
u \geq 0, \quad w \geq 0, \quad 0 \leq r \leq e
\end{array}
$$

onde $\bar{z}=f_{i_{p}}(\bar{x})$.

De (3.18) temos que

$$
z=\bar{z}+\nabla f_{i}(\bar{x})^{T}(x-\bar{x})+u_{i}-\bar{u}_{i}-w_{i}+\bar{w}_{i}, \quad i=1, \ldots, m
$$

De (3.17) segue que $e^{T} r=p$.

A igualdade (3.15) é válida se e somente se $\bar{w}_{i} r_{i}=0$ e $\bar{r}_{i} w_{i}=0$, que implica em 


$$
\begin{array}{r}
r_{i}=0, \quad i \in G(\bar{x}) \\
w_{i}=0, \quad i \in E^{1}(\bar{x}) \cup E^{*}(\bar{x}) \cup L(\bar{x})
\end{array}
$$

pois $w \geq 0, \bar{w} \geq 0, r \in[0,1]$ e $\bar{r} \in[0,1]$.

Como $\bar{y} \in \Gamma$, segue que

$$
\bar{u}^{T}(e-\bar{r})=0 \Rightarrow \bar{u}^{T} e=\bar{u}^{T} \bar{r}
$$

De (3.16) temos

$$
\begin{array}{r}
-\bar{u}^{T}(r-\bar{r})+(e-\bar{r})^{T}(u-\bar{u})=-\bar{u}^{T} r+\bar{u}^{T} \bar{r}+(e-\bar{r})^{T}(u-\bar{u}) \\
=\bar{u}^{T}(e-r)+u^{T}(e-\bar{r})=0
\end{array}
$$

Substituindo (3.21-3.23) em (3.20) temos:

1. Se $i \in G(\bar{x}) \cup E^{0}(\bar{x})$ então

$$
z=f_{i}(\bar{x})+\nabla f_{i}(\bar{x})^{T}(x-\bar{x})-w_{i}
$$

2. Se $i \in L(\bar{x}) \cup E^{1}(\bar{x})$ então

$$
z=f_{i}(\bar{x})+\nabla f_{i}(\bar{x})^{T}(x-\bar{x})+u_{i}
$$

3. Se $i \in E^{*}(\bar{x})$ então

$$
z=f_{i}(\bar{x})+\nabla f_{i}(\bar{x})^{T}(x-\bar{x})
$$

o que completa a prova.

Observe que o fato da componente $r$ poder assumir diferentes valores viáveis, dependendo dos indices $s, p$ e $l$ do ordenamento 3.3 , faz com que tenhamos, para cada $r$, um respectivo plano tangente $\Pi(y)$. Felizmente não teremos infinitos planos tangentes passíveis de análise, pois veremos que a 
escolha de $r$ tal que $r_{i} \in\{0,1\}$ já nos fornece toda informação necessária para encontrar direções de descida, se existirem. Além disso, a escolha $r_{i} \in(0,1)$ pode gerar planos tangentes formados por um único ponto, e portanto inúteis para projeção.

Sendo assim, temos o número exato de possíveis valores para a restauração do vetor $r$. De acordo com a notação pré-estabelecida para o ordenamento (3.3), a restauração de $r$ limita-se a atribuir valores $r_{i}=0$ e $r_{i}=1$ para $i \in i_{s+1}, \ldots, i_{l}$ de forma a valer

$$
\sum_{i \in\left\{i_{s+1}, \ldots, i_{\ell}\right\}} r_{i}=p-s
$$

já que, de (3.7), $r_{i}=1$ para $i=i_{1}, \ldots, i_{s}$. Logo, o número de restaurações de $r$ a ser analisado, $N_{r}$, é a combinação

$$
N_{r}=\left(\begin{array}{l}
l-s \\
p-s
\end{array}\right)
$$

Baseado na possibilidade de existência de mais de um vetor $r$ restaurado, definiremos o seguinte conjunto

$$
E^{+}(\bar{x}, r)=\left\{i \in E(\bar{x}) \mid r_{i}>0,(\bar{z}, \bar{x}, r, \bar{u}, \bar{w}) \text { é viável de }(3.2)\right\}
$$

Lembrando que a função objetivo de OVO* é dada por $g(y)=z$, dado $\bar{y}$ viável de (3.2), estamos interessados em projetar o ponto $y=\bar{y}-\nabla g(\bar{y})=$ $\bar{y}-e_{z}$ no plano tangente $\Pi(\bar{y})$, onde $e_{z}=(1,0, \ldots, 0)$. Denote esta projeção por $\tilde{y}=(\tilde{z}, \tilde{x}, \tilde{r}, \tilde{u}, \tilde{w}) \in \Pi(\bar{y})$, ou seja,

$$
\tilde{y}=P_{\Pi(\bar{y})}\left\{\bar{y}-e_{z}\right\}
$$

Portanto $\tilde{y}$ é solução do seguinte problema:

$$
\text { Minimizar }\left\|y-\bar{y}+e_{z}\right\| \quad \text { s.a. } \quad y \in \Pi(\bar{y})
$$

ou, equivalentemente,

$$
\text { Minimizar } h(y) \text { s.a. } y \in \Pi(\bar{y})
$$

onde

$$
h(y)=(z-\bar{z}+1)^{2}+\sum_{i=1}^{n}\left(x_{i}-\bar{x}_{i}\right)^{2}+\sum_{i=1}^{m}\left(r_{i}-\bar{r}_{i}\right)^{2}
$$




$$
+\sum_{i=1}^{m}\left(u_{i}-\bar{u}_{i}\right)^{2}+\sum_{i=1}^{m}\left(w_{i}-\bar{w}_{i}\right)^{2}
$$

Da caracterização do conjunto tangente dada no lema 3.4.1, sabemos que

$$
\begin{aligned}
\tilde{w}_{i} & =0 \quad i \in E^{+}(\bar{x}, r), \\
\nabla f_{i}(\bar{x})^{T}(\tilde{x}-\bar{x}) & =-\tilde{u}_{i}+(\tilde{z}-\bar{z}), \quad i \in E^{+}(\bar{x}, r) .
\end{aligned}
$$

Como $\bar{y} \in \Pi(\bar{y}), y=\bar{y}$ é um ponto viável para o problema (3.25), com valor da função objetivo $g(y)=1$.

Mas se qualquer outro ponto viável $y=(z, x, r, u, w)$ para o problema (3.25) satisfizer $z>\bar{z}$, então $z-\bar{z}+1>1 \mathrm{e}$, portanto, $y$ não é solução do problema, já que $h(y)>1$. Logo concluímos que a solução do problema de projeção deve satisfazer $z<\bar{z}$. Com isso, da igualdade (3.27), segue que

$$
\begin{gathered}
0>\tilde{z}-\bar{z}=\nabla f_{i}(\bar{x})^{T}(\tilde{x}-\bar{x})+\tilde{u}_{i} \geq \nabla f_{i}(\bar{x})^{T}(\tilde{x}-\bar{x}) \\
\Rightarrow \nabla f_{i}(\bar{x})^{T}(\tilde{x}-\bar{x})<0, \quad \forall i \in E^{+}(\bar{x}, r)
\end{gathered}
$$

Estes cálculos indicam que se não existir uma direção de descida das funções pertencentes a algum conjunto $E^{+}(\bar{x}, r)$, então a projeção será o próprio ponto em questão. Ou seja, $\bar{x}$ é um ponto estacionário, de acordo com a definição 3.2.3.

Essa direção de descida, se existir, depende da escolha da variável $r$, que pode assumir infinitos valores se procurarmos pontos viáveis $r \in \mathbb{R}^{m}$ satisfazendo $\left\{r_{i} \in[0,1], i \in E(\bar{x})\right.$ e $\left.\sum_{i \in E} r_{i}=p-s\right\}$. Felizmente, basta escolher $r_{i} \in\{0,1\}$ para encontrar, se existir, tal direção de descida. Para confirmarmos tal afirmação, defina o conjunto

$$
E_{\{0,1\}}(\bar{x}, r)=\left\{i \in E(\bar{x}) \mid r_{i}=1,(\bar{z}, \bar{x}, r, \bar{u}, \bar{w}) \text { é viável de (3.2) }\right\} .
$$

É fácil ver que $E_{\{0,1\}}(\bar{x}, r) \subseteq E^{+}(\bar{x}, r)$.

Lema 3.4.2. Se $\exists d \in \mathbb{R}^{n}$ tal que $\nabla f_{i}(\bar{x})^{T} d<0, \forall i \in E^{+}(\bar{x}, r)$ então $\exists E_{\{0,1\}}(\bar{x}, r)$ tal que $\nabla f_{i}(\bar{x})^{T} d<0, \forall i \in E_{\{0,1\}}(\bar{x}, r)$.

Prova: Basta redefinir o valor das componentes $i \in E^{+}(\bar{x}, r)$ de forma que $r_{i} \in\{0,1\}$ e $\sum_{i \in E^{+}} r_{i}=p-s$. O conjunto dos novos indices $i \in E(\bar{x})$ tal que $r_{i}=1$ formam o conjunto $E_{\{0,1\}}(\bar{x}, r)$.

O conjunto $E^{+}(\bar{x}, r)$ é de fundamental importância na projeção. Como já dissemos, o plano tangente depende da variável $r$. Por isso, baseado 
no lema 3.4.2, basta considerar $r_{i} \in\{0,1\}$. Mesmo tomando este cuidado podem existir conjuntos $E^{+}(\bar{x}, r)$ que possibilitam a obtenção de pontos estacionários indesejáveis, como é o caso do seguinte exemplo.

Exemplo: Seja $f_{1}(x)=x, f_{2}(x)=2 x, f_{3}(x)=x^{2}$, onde queremos minimizar a função OVO de ordem 2, representada por $f(x)$.Evidentemente, $x=0$ não é um "bom" ponto estacionário, pois não é nem máximo nem mínimo local de $f(x)$. Se a escolha de $r$ é tal que $r_{3}=0$ então teremos uma direção de descida, mas se $r_{3}=0$ então $x=0$ será um ponto estacionário (veja Figura 3.4).

\subsubsection{Explorando o conjunto tangente}

Nossa intenção aqui é interpretar o conjunto tangente às restrições de OVO* no espaço das variáveis do problema original OVO.

Para simplificar a notação, quando não houver dúvida quanto ao ponto $x$ usado, denotaremos os conjuntos $E(x), L(x), G(x), E^{1}(x), E^{0}(x)$ e $E^{*}(x)$, por $E, L, G, E^{1}, E^{0}$ e $E^{*}$, respectivamente.

A projeção de $\Pi(\bar{y})$ no eixo $(x, z)$ é dada pelo seguinte conjunto:

$\pi(\bar{x})=\left\{\begin{array}{ll} & z \geq f_{i}(\bar{x})+\nabla f_{i}(\bar{x})^{T}(x-\bar{x}), \quad i \in L(\bar{x}) \cup E^{1}(x) \\ (x, z) \in \Omega \times \Re \mid \quad z \leq f_{i}(\bar{x})+\nabla f_{i}(\bar{x})^{T}(x-\bar{x}), \quad i \in G(\bar{x}) \cup E^{0}(x) \\ z=f_{i}(\bar{x})+\nabla f_{i}(\bar{x})^{T}(x-\bar{x}), \quad i \in E^{*}(\bar{x})\end{array}\right\}$

Observando $\pi($.$) , no caso onde há empates é conveniente escolher E^{*}=$ $\varnothing$, pois esse seria o primeiro passo para evitar casos de planos tangentes degenerados, ou seja, $\pi(\bar{x})=\left(\bar{x}, f_{i_{p}}(\bar{x})\right)$. Entretanto essa escolha não é suficiente para evitar degeneração, como veremos nos exemplos a seguir. Este é o motivo pelo qual devemos considerar os $N_{r}$ possíveis valores da variável $r \in E^{+}(x, r)$, dado por $N_{r}=\left(\begin{array}{c}l-s \\ p-s\end{array}\right)$, no momento de resolver o problema de projeção (3.25), pois poderíamos estar projetando sobre um único ponto.

Exemplo 1. Considere as funções

$$
f_{1}(x)=x, f_{2}(x)=-x+2, \quad f_{3}(x)=0.5 x+2
$$

e $p=2$. Analisaremos qual é o conjunto $\pi(\bar{x})$ em determinados pontos viáveis $\bar{x}$. 
- $\bar{x}=2$ :

$$
\begin{aligned}
f(\bar{x}) & =f_{1}(\bar{x}) \\
L(\bar{x}) & =\{2\} \Rightarrow \bar{r}_{2}=1 \\
G(\bar{x}) & =\{3\} \Rightarrow \bar{r}_{3}=0 \\
E(\bar{x}) & =\{1\}
\end{aligned}
$$

Como $\bar{r}^{T} e=p=2$, segue que $\bar{r}_{1}=1$. Logo $E^{1}=\{1\}$ e $E^{0}=\emptyset$.

Pelo lema 3.4.1, o poliedro é dado por

$$
\pi(\bar{x})=\left\{(x, z) \in \Omega \times \Re \mid \begin{array}{l}
z \geq-x+2 \\
z \leq 0.5 x+2 \\
z \geq x
\end{array}\right\}
$$

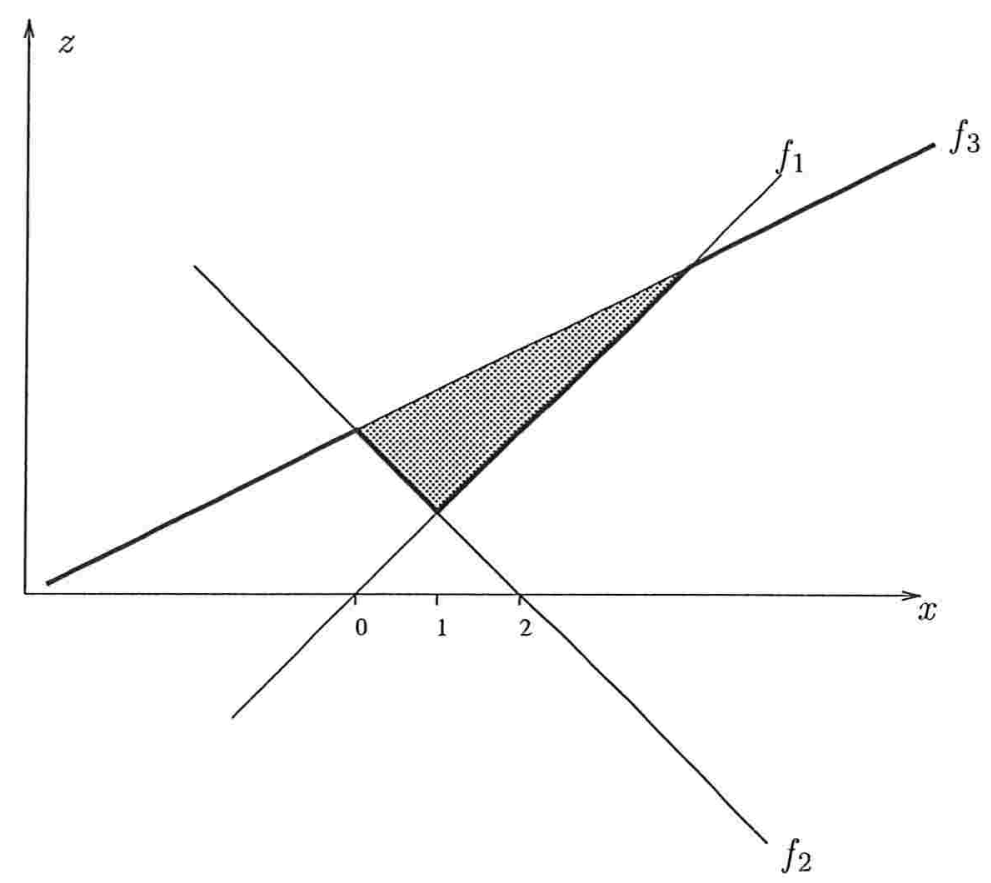

Figura 3.2: Plano tangente no caso de não haver empate $(\bar{x}=2)$.

Esta região está representada na figura 3.2. 
- $\bar{x}=1$ :

$$
\begin{aligned}
f(\bar{x}) & =f_{1}(\bar{x})=f_{2}(\bar{x}) \Rightarrow E(\bar{x})=\{1,2\} \\
L(\bar{x}) & =\emptyset \\
G(\bar{x}) & =\{3\} \Rightarrow \bar{r}_{3}=0 \\
e^{T} \bar{r} & =2 \Rightarrow E^{1}(\bar{x})=\{1,2\}, \quad E^{0}(\bar{x})=\emptyset
\end{aligned}
$$

Neste caso, $\bar{r}=[1,1,0]$ e a figura representando $\pi(\bar{x})$ é idêntica à figura 3.2 .

- $\bar{x}=0$ :

$$
\begin{array}{r}
f(\bar{x})=f_{2}(\bar{x})=f_{3}(\bar{x}) \\
G(\bar{x})=\emptyset, \quad E(\bar{x})=\{2,3\} \\
L(\bar{x})=\{1\} \Rightarrow \bar{r}_{1}=1
\end{array}
$$

Neste caso há três possibilidades de escolha para $\bar{r}$ :

a. $\bar{r}=[1,0,1]$

Neste caso, $E^{0}(\bar{x})=\{2\}, \quad E^{1}(\bar{x})=\{3\}$

O conjunto $\pi(\bar{x})$ é dado por

$$
\pi(\bar{x})=\left\{(x, z) \in \Omega \times \Re \mid \begin{array}{l}
z \leq-x+2 \\
z \geq 0.5 x+2 \\
z \geq x
\end{array}\right\}
$$

O respectivo conjunto tangente está representado na figura 3.3 .

b. $\bar{r}=[1,1,0]$

Neste caso $E^{0}(\bar{x})=\{3\}, \quad E^{1}(\bar{x})=\{2\}$

O conjunto $\pi(\bar{x})$ é dado por

$$
L(\bar{x})=\left\{(x, z) \in \Omega \times \Re \mid \begin{array}{l}
z \geq-x+2 \\
z \leq 0.5 x+2 \\
z \geq x
\end{array}\right\}
$$

A figura do conjunto tangente é idêntica a figura 3.2 . 


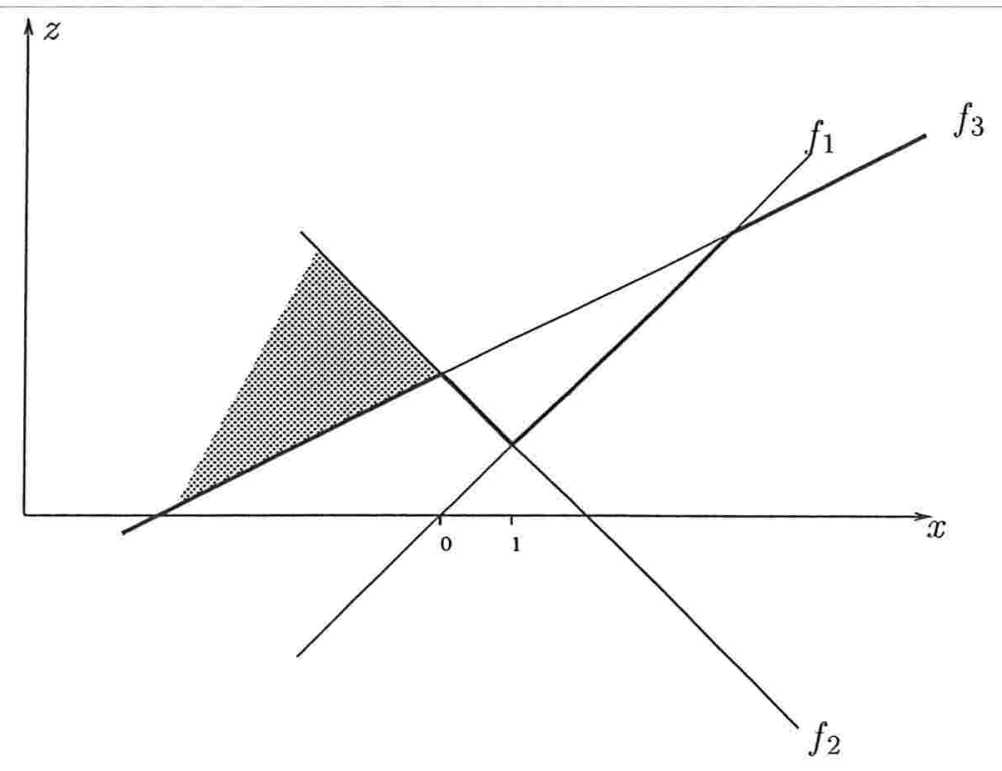

Figura 3.3: Plano tangente no caso de haver empate $(\bar{x}=0)$, com $\bar{r}=$ $[1,0,1]$.

c. $\bar{r}=[1, a, 1-a], a \in(0,1)$

Neste caso $E^{*}(\bar{x})=\{2,3\}, \quad E^{1}(\bar{x})=\emptyset, \quad E^{0}(\bar{x})=\emptyset$

O conjunto $\pi(\bar{x})$ é dado por

$$
\pi(\bar{x})=\left\{(x, z) \in \Omega \times \Re \mid \begin{array}{l}
z=-x+2 \\
z=0.5 x+2 \\
z \geq x
\end{array}\right\}=\{(0,2)\}
$$

Neste caso, o conjunto $\pi(\bar{x})$ é degenerado.

Os traços em negrito nas figuras representam a função objetivo $f(x)$.

Exemplo 2: Considere as funções

$$
f_{1}(x)=x, f_{2}(x)=2 x, \quad f_{3}(x)=x^{2}
$$

e $p=2$. Se $\bar{x}=0$, temos $E^{1}=\{1,2,3\}, \quad L(\bar{x})=G(\bar{x})=\emptyset$. Há 3 possibilidades de escolha para $L(\bar{x})$ :

- $\bar{r}=[1,1,0] \Rightarrow L(0)=$ região $A$ do gráfico 3.4 ; 
- $\bar{r}=[1,0,1] \Rightarrow L(0)=$ região $B$ do gráfico 3.4 ;

- $\bar{r}=[0,1,1] \Rightarrow L(0)=\{(0,0)\}$.

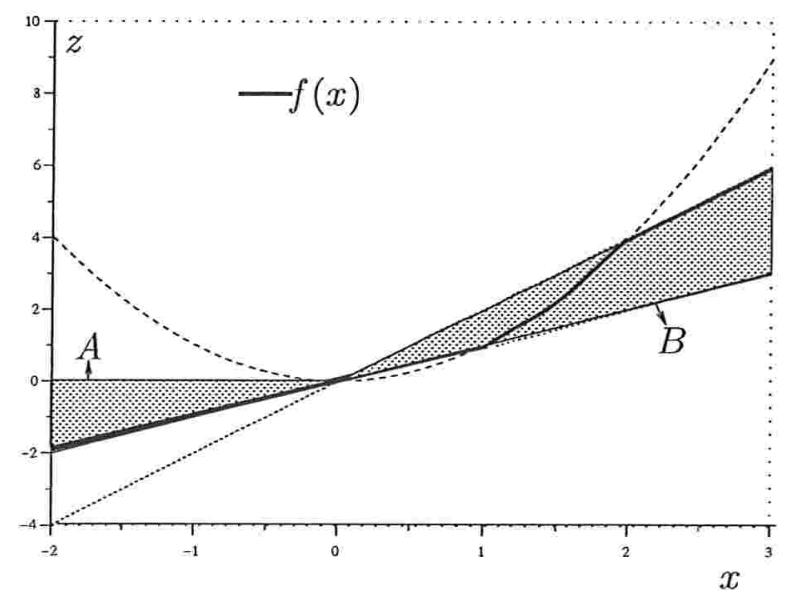

Figura 3.4: Plano tangente no caso de haver empate $(\bar{x}=0)$.

\subsection{Gradiente Projetado Restaurado}

Nesta seção sugerimos um algoritmo para resolver OVO* usando uma sequência de passos de projeção e restauração exata do gradiente, com a condição de otimalidade dada por 3.2.1.

Nas seções anteriores caracterizamos o poliedro definido pelo hiperplano tangente as restrições de $\mathrm{OVO}^{*}$ mais as restrições de caixa $\Gamma$, denotado por $\Pi(\bar{y})$. Após projetarmos o vetor $\bar{y}-e_{z}$ em $\Pi(\bar{y})$ obtemos $\tilde{y}$, que não necessariamente é viável para o problema $\mathrm{OVO}^{*}$. Neste caso, podemos restaurá-lo usando (3.4)-(3.7), como vemos na Figura 3.5.

Um critério de parada para tal algoritmo poderia ser verificar se a projeção é o mesmo ponto $\bar{y}$, significando que a condição de otimalidade dada em 3.2.1 estaria satisfeita.

Algoritmo (Método do Gradiente Projetado Restaurado)

Encontre um ponto inicial $x^{0} \in \Omega$; 


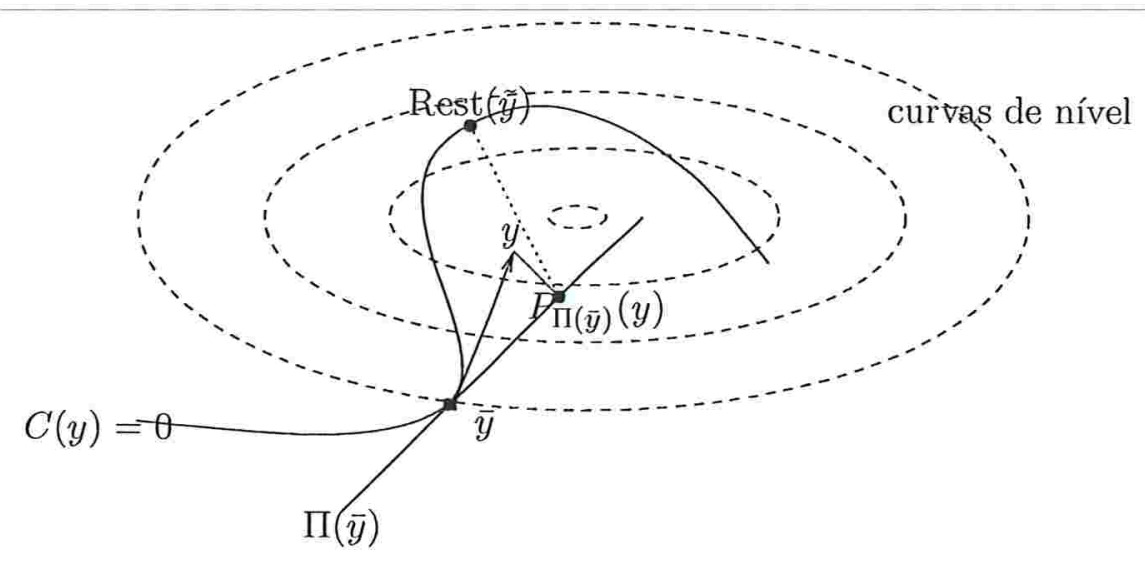

Figura 3.5: Passos do gradiente projetado restaurado.

for $k=0,1, \ldots$

Defina $d=0$.

Restaure $x^{k}$ usando $3.4-3.7$, obtendo $\left(z^{k}, x^{k}, u^{k}, w^{k}\right)$;

while não achar um valor de $r^{k}$ adequado, faça:

Projete $y^{k}-e_{z}$ no hiperplano $\Pi\left(y^{k}\right)$, obtendo o ponto $\tilde{y}=$ $(\tilde{z}, \tilde{x}, \tilde{r}, \tilde{u}, \tilde{w})$

Se $\tilde{z} \geq z^{k}$, então $y^{k}$ é solução provisória. Tente outro $r^{k}$, se houver. Senão pare;

Se $\tilde{z}<z^{k}$ então a direção $d=\tilde{y}-y^{k}$ é uma direção de descida de $z$

fim (while)

Se $d=0$ pare. Retorne $y^{k}$ como solução.

Senão escolha $y=y^{k}+\alpha d$ viável para 3.2 , tal que $f_{p}(x)<$ $f_{p}\left(x^{k}\right)$

$x^{k+1} \leftarrow x$.

end (for)

A dificuldade neste tipo de algoritmo reside na tarefa de projetar um vetor em um conjunto como $\Pi(y)$. Observe que a intenção aqui é somente deixar o tema "gradiente projetado" como uma possível linha de estudo para atacar o problema 3.1, seguindo as idéias gerais de Rom e Avriel nos trabalhos [13] e [14]. 


\section{Capítulo 4}

\section{OVO e o Problema de Desigualdade Variacional}

Neste capítulo exploramos uma equivalência entre o problema OVO* e um problema de desigualdade variacional, conhecido como VIP. O propósito desta relação é reduzir um problema de desigualdade variacional a um problema de otimização com função objetivo diferenciável e restrições de caixa. $\mathrm{Na}$ seção 1 definimos o problema de desigualdade variacional geral. Na seção 2 apresentamos um resultado teórico importante, estabelecendo condições para que pontos estacionários de um PNL sejam soluções do VIP. Na seção 3 veremos que o problema $\mathrm{OVO}^{*}$ satisfaz essas condições e, portanto, podemos resolvê-lo através de algoritmos já conhecidos da literatura para PNL.

\subsection{Introdução}

Problemas de complementaridade passaram a ser alvo de muita pesquisa principalmente após o aparecimento das condições de otimalidade para problemas não lineares de otimização contínua, envolvendo restrições de desigualdade. A princípio, o estudo do problema de complementaridade linear (LCP) derivou das condições de otimalidade de KKT para problemas lineares e quadráticos. Depois foram introduzidos os problemas de complementaridade não linear (NCP), por Cottle [5], e posteriormente os problemas de desigualdade variacional (VIP), por Hartman e Stampacchia [7]. Com o intuito de introduzir o leitor no assunto, definiremos os problemas aqui mencionados, dando ênfase posteriormente ao estudo de um específico VIP, relacionado ao problema OVO.

O caso mais simples de LCP pode ser descrito da seguinte forma: dados 
$M \in \mathbb{R}^{n \times n}, q \in \mathbb{R}^{n}$, encontre vetores $w$ e $z$ de $\mathbb{R}^{n}$ satisfazendo

$$
w-M z=q, \quad w \geq 0, \quad z \geq 0, \quad w^{T} z=0 .
$$

Denotamos este problema por $\operatorname{LCP}(q, M)$. Este problema é dito ser monótono se a matriz $M$ for semidefinida positiva.

Uma primeira generalização de $L C P$ é o chamado $L C P$ misto, ou $M L C P$ : dados $A \in \mathbb{R}^{n \times n}, B \in \mathbb{R}^{m \times m}, C \in \mathbb{R}^{n \times m}, D \in \mathbb{R}^{m \times n}, a \in \mathbb{R}^{n}, b \in \mathbb{R}^{m}$, encontre $u \in \mathbb{R}^{n}$ e $v \in \mathbb{R}^{m}$ tais que

$$
\begin{gathered}
a+A u+C v=0 \\
b+D u+B v \geq 0, \quad v \geq 0 \\
v^{T}(b+D u+B v)=0 .
\end{gathered}
$$

Este problema é um misto de LCP e um sistema de equações lineares com variáveis livres de sinal.

Agora definiremos duas generalizações não lineares de (4.1). A primeira é conhecida como NCP: dado o mapeamento $F(y)=\left(F_{i}(y)\right): \mathbb{R}^{n} \rightarrow \mathbb{R}^{n}$, encontre um vetor $y \in \mathbb{R}^{n}$ satisfazendo

$$
y \geq 0, \quad F(y) \geq 0, \quad y^{T} F(z)=0 .
$$

Se $F$ é função afim $q+M y$, então (4.3) torna-se o $L C P(q, M)$.

Uma generalização mais complexa de (4.3) é o problema de desigualdade variacional: dado o mapeamento $F(y)=\left(F_{i}(y)\right): \mathbb{R}^{n} \rightarrow \mathbb{R}^{n} \mathrm{e} \emptyset \neq K \subset \mathbb{R}^{n}$, encontre um vetor $x^{*} \in K$ tal que

$$
\left(y-x^{*}\right)^{T} F\left(x^{*}\right) \geq 0, \quad \text { para todo } y \in K .
$$

denotado por $\operatorname{VIP}(K, F)$. Se $K=\{z: z \geq 0\}$, então o vetor $x^{*}$ que resolve (4.3) também resolve (4.4). Além disso, se $K$ é um poliedro e $F$ é uma função afim, pode-se provar que $\operatorname{VIP}(K, F)$ é um particular LCP. Quando $K$ é definido por caixas $\Pi_{i=1}^{n}\left[l_{i}, u_{i}\right],-\infty \leq l_{i}<u_{i} \leq \infty, i=1, \ldots, n$, o problema é conhecido como como um problema de complementaridade (não linear) misto, chamado de $M C P$.

Uma estratégia atrativa para resolver o VIP é usar reformulações baseadas na transformação deste em um problema de otimização global. Como a maioria dos algoritmos usados para resolver problemas de programação não lineares encontram pontos estacionários, é natural perguntar em que condições podemos garantir que um ponto estacionário, solução do PNL, resolve também o VIP. É o que faremos na próxima seção para um específico $M C P$, que nos será útil na resolução do problema OVO. 


\subsection{Um resultado para VIP}

Defina o conjunto $\mathcal{B}$ por

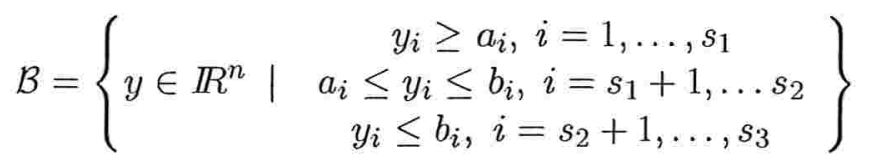

Considere o VIP definido pelo operador linear $F(y)=M y+q$ no conjunto $\mathcal{B}$. Na notação já inserida, este VIP será denotado por $\operatorname{VIP}(M y+q, \mathcal{B})$. Dizemos que o VIP é viável se existe $y \in \mathcal{B}$ tal que

$$
\begin{aligned}
& {[M y+q]_{i} \geq 0, \quad \text { se } i=1, \ldots s_{1}} \\
& {[M y+q]_{i} \leq 0, \quad \text { se } i=s_{2}+1, \ldots s_{3}} \\
& {[M y+q]_{i}=0, \quad \text { se } i=s_{3}+1, \ldots n}
\end{aligned}
$$

onde

$$
M=\left[\begin{array}{c}
m_{1}^{T} \\
m_{2}^{T} \\
\vdots \\
m_{n}^{T}
\end{array}\right]
$$

Defina a função $R$ por:

$$
\begin{aligned}
R(y, \varrho, \beta) & =\sum_{i=1}^{s_{1}}\left(m_{i}^{T} y+q_{i}-\varrho_{i}\right)^{2}+\sum_{i=s_{1}+1}^{s_{2}}\left(m_{i}^{T} y+q_{i}-\varrho_{i}+\beta_{i}\right)^{2} \\
& +\sum_{i=s_{2}+1}^{s_{3}}\left(m_{i}^{T} y+q_{i}+\beta_{i}\right)^{2}+\sum_{i=s_{3}+1}^{n}\left(m_{i}^{T} y+q_{i}\right)^{2} \\
& +\left(\sum_{i=1}^{s_{2}}\left(y_{i}-a_{i}\right)^{\nu_{1}} \varrho_{i}^{\nu_{1}}\right)^{\nu_{2}}+\left(\sum_{i=s_{1}+1}^{s_{3}}\left(b_{i}-y_{i}\right)^{\nu_{1}} \beta_{i}^{\nu_{1}}\right)^{\nu_{2}} .
\end{aligned}
$$

Considere o seguinte problema de otimização:

$$
\operatorname{Min} R(y, \varrho, \beta) \text { s.t. } y \in \mathcal{B}, \varrho \geq 0, \beta \geq 0 .
$$

Relembremos que uma matriz $A \in R^{n \times n}$ é coluna-suficiente (ou "columnsufficient" ) (veja [5]) se para todo $u \in R^{n}$ tal que

$$
[u]_{i}[A u]_{i} \geq 0 \text { para todo } i=1, \ldots, n
$$


temos necessariamente que

$$
[u]_{i}[A u]_{i}=0 \text { para todo } i=1, \ldots, n .
$$

Uma matriz $A$ é linha-suficiente (ou "row-sufficient") se $A^{T}$ é colunasuficiente (ou "column-sufficient"), e $A$ é suficiente (ou "sufficient") se ela é tanto linha-suficiente como coluna-suficiente.

Toda matriz semidefinida positiva é linha-suficiente, mas o oposto não é verdade (ver [5]). Por outro lado, um operador linear é monótono se, e somente se, sua matriz Jacobiana é semidefinida positiva.

Teorema 4.2.1. Se $M$ é linha-suficiente, se o problema VIP $(M y+q, \mathcal{B})$ é viável, $\nu_{1} \geq 1, \nu_{2} \geq 1, \nu_{1}+\nu_{2}>2$, então todo ponto estacionário de (4.6) é uma solução de VIP $(M y+q, \mathcal{B})$.

Prova. Defina $\xi \in \mathbb{R}^{n}, \theta_{1}$ e $\theta_{2}$ tais que

$$
\begin{aligned}
& \xi_{i}=\left\{\begin{array}{cl}
m_{i}^{T} y+q_{i}-\varrho_{i} & \text { se } i=1, \ldots, s_{1} \\
m_{i}^{T} y+q_{i}-\varrho_{i}+\beta_{i} & \text { se } i=s_{1}+1, \ldots, s_{2} \\
m_{i}^{T} y+q_{i}+\beta_{i} & \text { se } i=s_{2}+1, \ldots, s_{3} \\
m_{i}^{T} y+q_{i} & \text { caso contrário }
\end{array}\right. \\
& \theta_{1}=\sum_{i=1}^{s_{2}}\left(y_{i}-a_{i}\right)^{\nu_{1}} \varrho_{i}^{\nu_{1}} \\
& \theta_{2}=\sum_{i=s_{1}+1}^{s_{3}}\left(b_{i}-y_{i}\right)^{\nu_{1}} \beta_{i}^{\nu_{1}},
\end{aligned}
$$

As condições KKT de (4.6) são as seguintes:

$$
\begin{array}{cl}
2\left[M^{T} \xi\right]_{i}+\nu_{2} \nu_{1} \theta_{1}^{\nu_{2}-1}\left(y_{i}-a_{i}\right)^{\nu_{1}-1} \varrho_{i}^{\nu_{1}}-\mu_{i}=0 & \text { se } i=1, \ldots, s_{1} \\
2\left[M^{T} \xi\right]_{i}+\nu_{2} \nu_{1} \theta_{1}^{\nu_{2}-1}\left(y_{i}-a_{i}\right)^{\nu_{1}-1} \varrho_{i}^{\nu_{1}} & \\
-\nu_{2} \theta_{2}^{\nu_{2}-1}\left(b_{i}-y_{i}\right)^{\nu_{1}-1} \beta_{i}^{\nu_{1}} \nu_{1}-\mu_{i}+\gamma_{i}=0 & \text { se } i=s_{1}+1, \ldots, s_{2} \\
2\left[M^{T} \xi\right]_{i}-\nu_{2} \nu_{1} \theta_{2}^{\nu_{2}-1}\left(b_{i}-y_{i}\right)^{\nu_{1}-1} \beta_{i}^{\nu_{1}}+\gamma_{i}=0 & \text { se } i=s_{2}+1, \ldots, s_{3} \\
2\left[M^{T} \xi\right]_{i}=0 & \text { se } i=s_{3}+1, \ldots, n \\
-2 \xi_{i}+\nu_{2} \nu_{1} \theta_{1}^{\nu_{2}-1}\left(y_{i}-a_{i}\right)^{\nu_{1}} \varrho_{i}^{\nu_{1}-1}-\lambda_{i}=0, & \text { se } i=1, \ldots, s_{2}
\end{array}
$$




$$
\begin{gathered}
2 \xi_{i}+\nu_{2} \nu_{1} \theta_{2}^{\nu_{2}-1}\left(b_{i}-y_{i}\right)^{\nu_{1}} \beta_{i}^{\nu_{1}-1}-\pi_{i}=0, \text { se } i=s_{1}+1, \ldots, s_{3} \\
\left(y_{i}-a_{i}\right) \mu_{i}=\varrho_{i} \lambda_{i}=0 \text { se } i=1, \ldots, s_{2} \\
\left(b_{i}-y_{i}\right) \gamma_{i}=\beta_{i} \pi_{i}=0 \text { se } i=s_{1}+1, \ldots, s_{3} \\
y \in B, \varrho \geq 0, \beta \geq 0, \lambda \geq 0, \pi \geq 0, \mu \geq 0, \gamma \geq 0 .
\end{gathered}
$$

(I) Para $i=1, \ldots, s_{1}$

Por (4.8) temos:

$$
2 \xi_{i}=\nu_{2} \nu_{1} \theta_{1}^{\nu_{2}-1}\left(y_{i}-a_{i}\right)^{\nu_{1}} \varrho_{i}^{\nu_{1}-1}-\lambda_{i} .
$$

Por (4.7) e (4.10), premultiplicando por $2 \xi_{i}$, obtemos:

$$
4 \xi_{i}\left[M^{T} \xi\right]_{i}+\left(\nu_{2} \nu_{1}\right)^{2} \theta_{1}^{2 \nu_{2}-2}\left(\left(y_{i}-a_{i}\right) \varrho_{i}\right)^{2 \nu_{1}-1}+\lambda_{i} \mu_{i}=0 .
$$

(II) Para $i=s_{2}+1, \ldots, s_{3}$

Por (4.9) temos:

$$
2 \xi_{i}=-\nu_{2} \nu_{1} \theta_{2}^{\nu_{2}-1}\left(b_{i}-y_{i}\right)^{\nu_{1}} \beta_{i}^{\nu_{1}-1}+\pi_{i}
$$

De (4.7) e (4.11), premultiplicando por $2 \xi_{i}$, obtemos:

$$
4 \xi_{i}\left[M^{T} \xi\right]_{i}+\left(\nu_{2} \nu_{1}\right)^{2} \theta_{2}^{2 \nu_{2}-2}\left(\left(b_{i}-y_{i}\right) \beta_{i}\right)^{2 \nu_{1}-1}+\gamma_{i} \pi_{i}=0,
$$

(III) Para $i=s_{1}+1, \ldots, s_{2}$

Como em (4.8), por (4.9)

$2 \xi_{i}=-\nu_{2} \nu_{1} \theta_{2}^{\nu_{2}-1}\left(b_{i}-y_{i}\right)^{\nu_{1}} \beta_{i}^{\nu_{1}-1}+\pi_{i}=\nu_{2} \nu_{1} \theta_{1}^{\nu_{2}-1}\left(y_{i}-a_{i}\right)^{\nu_{1}} \varrho_{i}^{\nu_{1}-1}-\lambda_{i}$

Premultiplicando (4.7) por $2 \xi_{i}$, para $i=s_{1}+1, \ldots, s_{2}$, de $(4.10) \mathrm{e}$ (4.11) temos:

$$
\begin{gathered}
4 \xi_{i}\left[M^{T} \xi\right]_{i}+\left(\nu_{2} \nu_{1}\right)^{2} \theta_{1}^{2 \nu_{2}-2}\left(\left(y_{i}-a_{i}\right) \varrho_{i}\right)^{2 \nu_{1}-1}+ \\
\left(\nu_{1} \nu_{2}\right)^{2} \theta_{2}^{2 \nu_{2}-2}\left(\left(b_{i}-y_{i}\right) \beta_{i}\right)^{2 \nu_{1}-1}+\lambda_{i} \mu_{i}+\gamma_{i} \pi_{i}=0
\end{gathered}
$$


(IV) Para $i=s_{3}, \ldots, n$

Analogamente, de (4.7), concluímos que

$$
4 \xi_{i}\left[M^{T} \xi\right]_{i}=0
$$

De (4.14),(4.16), (4.18) e (4.19), levando em conta os sinais das variáveis em (4.12), obtemos:

$$
\xi_{i}\left[M^{T} \xi\right]_{i} \leq 0 \text { se } i=1, \ldots, n
$$

De (4.20), e do fato da matriz ser linha suficiente, temos:

$$
\xi_{i}\left[M^{T} \xi\right]_{i}=0 \text { se } i=1, \ldots, n
$$

Lembrando que $\nu_{1} \geq 1, \nu_{2} \geq 1$ concluímos que

$$
\theta_{1}=\theta_{2}=0
$$

Desde que $\nu_{1}+\nu_{2}>2$, as condições de KKT podem ser escritas da seguinte forma:

$$
\begin{array}{cl}
2\left[M^{T} \xi\right]_{i}-\mu_{i}=0 & \text { se } i=1, \ldots, s_{1} \\
2\left[M^{T} \xi\right]_{i}-\mu_{i}+\gamma_{i}=0 & \text { se }=i=s_{1}+1, \ldots, s_{2} \\
2\left[M^{T} \xi\right]_{i}+\gamma_{i}=0 & \text { se } i=s_{2}+1, \ldots, s_{3} \\
2\left[M^{T} \xi\right]_{i}=0 & \text { se } i=s_{3}+1, \ldots, n \\
-2 \xi_{i}-\lambda_{i}=0, & \text { se } i=1, \ldots, s_{2} \\
2 \xi_{i}-\pi_{i}=0, \text { se } i=s_{1}+1, \ldots, s_{3} \\
\left(y_{i}-a_{i}\right) \mu_{i}=\varrho_{i} \lambda_{i}=0 \text { se } i=1, \ldots, s_{2} \\
\left(b_{i}-y_{i}\right) \gamma_{i}=\beta_{i} \pi_{i}=0 \text { se } i=s_{1}+1, \ldots, s_{3} \\
y \in B, \varrho \geq 0, \beta \geq 0, \lambda \geq 0, \pi \geq 0, \mu \geq 0, \gamma \geq 0 .
\end{array}
$$

Mas (4.23)-(4.28) são as condições de KKT do problma convexo:

$$
\operatorname{Min} \sum_{i=1}^{s_{1}}\left(m_{i}^{T} y+q_{i}-\varrho_{i}\right)^{2}+\sum_{i=s_{1}+1}^{s_{2}}\left(m_{i}^{T} y+q_{i}-\varrho_{i}+\beta_{i}\right)^{2}
$$




$$
\begin{gathered}
+\sum_{i=s_{2}+1}^{s_{3}}\left(m_{i}^{T} y+q_{i}+\beta_{i}\right)^{2}+\sum_{i=s_{3}+1}^{n}\left(m_{i}^{T} x+q_{i}\right)^{2} \\
\text { s.t. } \quad y \in \mathcal{B}, \varrho \geq 0, \beta \geq 0 .
\end{gathered}
$$

Agora, pela viabilidade do problema, concluímos que $\xi=0$, o que completa a prova.

O teorema 4.2.1 nos permite resolver um problema de complementaridade misto do tipo $\operatorname{VIP}(M y+q, \mathcal{B})$, dado que as condições do teorema sejam satisfeitas, com qualquer algoritmo razoável de PNL aplicado ao problema (4.6), já que um ponto estacionário de (4.6) é uma solução de $\operatorname{VIP}(M y+q, \mathcal{B})$. Na proxima seção aplicaremos este resultado ao problema OVO.

\subsection{Extensão dos resultados ao problema OVO}

Nesta seção aplicaremos os resultados anteriores ao problema OVO. Em particular veremos que as restrições de $\mathrm{OVO}^{*}$ possuem propriedades que garantem as hipóteses do teorema 4.2.1.

Dado $x \in \Omega$, defina $F(x)=\left(f_{1}(x), \ldots, f_{m}(x)\right)^{T}$ e $T: \mathbb{R}^{m+m+1} \rightarrow$ $\mathbb{R}^{m+m+1}(T=T(r, u, z))$ por

$$
T(x ; r, u, z)=\left[\begin{array}{l}
u-z e+F(x) \\
e-r \\
\langle r, e\rangle-(p-1)
\end{array}\right] .
$$

Defina também $\Gamma \subset \mathbb{R}^{m+m+1}$ por

$$
\Gamma=\left\{(r, u, z) \in \mathbb{R}^{m+m+1} \mid r \geq 0, u \geq 0\right\} .
$$

(Por abuso de notação, escreveremos $(r, u, z)$ ao invés de $\left(r^{T}, u^{T}, z^{T}\right)^{T}$ quando não houver perigo de confusão). Dizemos que $(r, u, z)$ resolve o problema VIP associado ao operador $T$ e ao conjunto $\Gamma(\operatorname{VIP}(x, T, \Gamma)$ de agora em diante) se

$$
\left\langle T(r, u, z),\left(r^{\prime}-r, u^{\prime}-u, z^{\prime}-z\right)\right\rangle \geq 0 \text { para todo }\left(r^{\prime}, u^{\prime}, z^{\prime}\right) \in \Gamma .
$$

O conjunto de soluções $(r, u, z)$ do $\operatorname{VIP}(x, T, \Gamma)$ será chamado de $\operatorname{SOLVIP}(x, T, \Gamma)$.

O próximo teorema 4.3.2 nos mostra que as restrições de OVO* pode ser formuladas como a solução de um $\operatorname{VIP}(x, T, \Gamma)$. Antes, porém, segue um teorema já conhecido na literatura (ver [2]). 
Teorema 4.3.1. Sejam $F: \mathbb{R}^{n} \rightarrow \mathbb{R}^{n}, F(x)=\left[f_{1}(x), \ldots, f_{m}(x)\right], \Omega=$ $\left\{x \in \mathbb{R}^{n}: x_{i} \geq 0 \quad i=1, \ldots, p\right\}$. Então $x^{*} \in \Omega$ satisfaz

$$
F\left(x^{*}\right)^{T}\left(x-x^{*}\right) \geq 0, \quad \forall x \in \Omega
$$

se e somente se

$$
\begin{gathered}
f_{i}\left(x^{*}\right) \geq 0, \quad x_{i}^{*} f_{i}\left(x^{*}\right)=0, \quad i=1, \ldots, p \\
f_{i}\left(x^{*}\right)=0, \quad i=p+1, \ldots, m .
\end{gathered}
$$

Teorema 4.3.2. Dado $x \in \Omega,(r, u, z)$ satisfaz as restrições (2.9) se e somente se $(r, u, z)$ resolve $\operatorname{VIP}(x, T, \Gamma)$.

Prova. A prova segue imediantamente do teorema 4.3.1.

De acordo com o teorema 4.3.2, o problema OVO* pode ser reformulado como sendo o seguinte MPEC:

$$
\text { Minimize } z \text { s.t. }(r, u, z) \in \operatorname{SOLVIP}(x, T, \Gamma), x \in \Omega \text {. }
$$

Observe que o operador $T$ é linear para todo $x \in \Omega$ fixado. Seu Jacobiano $J_{T}(r, u, z)$ é dado por

$$
J_{T}(r, u, z)=\left[\begin{array}{ccc}
0 & I & e \\
-I & 0 & 0 \\
e^{T} & 0 & 0
\end{array}\right]
$$

Um cálculo direto nos mostra que $\left\langle J_{T}(r, u, z)(r, u, z),(r, u, z)\right\rangle=0$ para todo $(r, u, z) \in \mathbb{R}^{m+m+1}$. Portanto, o operador é monótono e, consequentemente, linha-suficiente, onde $M=J_{T}(r, u, z)$. O teorema 4.3 .2 nos garante que um ponto estacionário solução de (4.6) é solução de $\operatorname{VIP}(M y+q, \Gamma)$. Isto nos permite obter solução de OVO usando algoritmos para PNL aplicados ao problema (4.30). 


\section{Capítulo 5}

\section{Método Cauchy Primal para OVO}

\subsection{Introdução}

Relembrando, dadas $m$ funções contínuas $f_{1}, \ldots, f_{m}$, definidas em um domínio $\Omega \subset \mathbb{R}^{n}$, e um inteiro $p \in\{1, \ldots, m\}$, a função OVO de ordem $(p) f(x)$ é dada por

$$
f(x)=f_{i_{p}(x)}(x)
$$

onde

$$
f_{i_{1}(x)}(x) \leq f_{i_{2}(x)}(x) \leq \ldots \leq f_{i_{p}(x)}(x) \leq \ldots \leq f_{i_{m}(x)}(x) .
$$

Já vimos que $f$ é contínua. Entretanto, mesmo que as funções $f_{i}$ sejam diferenciáveis, a função OVO pode não ser diferenciável em alguns pontos.

O problema OVO consiste na minimização da função Order-Value:

$$
\text { Minimizar } f(x) \text { s.a. } x \in \Omega \text {. }
$$

que já vimos ser equivalente a

$$
\begin{aligned}
& \text { Minimizar } z \\
& \text { s.a. }\left\{\begin{array}{l}
\sum_{i=1}^{m} r_{i}\left(u_{i}-z+f_{i}(x)\right)=0 \\
\sum_{i=1}^{m}\left(1-r_{i}\right) u_{i}=0 \\
\sum_{i=1}^{m} r_{i}=p \\
u_{i}-z+f_{i}(x) \geq 0 \quad i=1, \ldots, m \\
u \geq 0, \quad 0 \leq r \leq e, \\
x \in \Omega .
\end{array}\right.
\end{aligned}
$$


A principal desvantagem desta abordagem é o aumento significativo do número de variáveis. Neste capítulo introduziremos um método "primal" para OVO, de forma que somente as variáveis $x$ estão envolvidas. O algoritmo será apresentado na seção 2 , onde um teorema de convergência é provado. Na seção 3 descrevemos uma maneira de resolver aproximadamente os subproblemas, satisfazendo as hipóteses teóricas.

\subsection{O algoritmo}

Suponha que $\Omega$ seja um conjunto fechado e convexo, e $f_{1}, \ldots, f_{m}$ tenham derivadas parciais contínuas em um conjunto aberto contendo $\Omega$. Denote, daqui por diante, $g_{j}=\nabla f_{j}$.

Para todo $x, y \in \Omega, j=1, \ldots, m$, assumiremos que

$$
\left\|g_{j}(x)\right\|_{\infty} \leq c
$$

$\mathrm{e}$

$$
\left\|g_{j}(y)-g_{j}(x)\right\|_{\infty} \leq L\|y-x\|_{\infty} .
$$

Consequentemente, para todo $x, y \in \Omega, j=1, \ldots, m$,

$$
\left|f_{j}(y)-f_{j}(x)\right| \leq c\|y-x\|_{\infty}
$$

e

$$
f_{j}(y) \leq f_{j}(x)+g_{j}(x)^{T}(y-x)+\frac{L}{2}\|y-x\|_{\infty}^{2} .
$$

Dado $\varepsilon \geq 0, x \in \Omega$, definimos

$$
I_{\varepsilon}(x)=\left\{j \in\{1, \ldots, m\} \mid f(x)-\varepsilon \leq f_{j}(x) \leq f(x)+\varepsilon\right\} .
$$

O teorema abaixo nos fornece uma condição necessária de otimalidade para o problema OVO.

Teorema 5.2.1. Se $x_{*} \in \Omega$ é um minimizador local de $f(x)$ sujeito a $x \in \Omega$ e $\varepsilon>0$, então

$$
\mathcal{D} \equiv\left\{d \in \mathbb{R}^{n} \mid x_{*}+d \in \Omega \text { e } g_{j}\left(x_{*}\right)^{T} d<0 \forall j \in I_{\varepsilon}\left(x_{*}\right)\right\}=\emptyset .
$$

Prova. Suponha, por absurdo, que (5.6) seja falso. Então, existe $d \in \mathbb{R}^{n} \mathrm{e}$ $\bar{\alpha}>0$ tais que $x_{*}+d \in \Omega \mathrm{e}$

$$
f_{j}\left(x_{*}+\alpha d\right)<f_{j}\left(x_{*}\right) \forall \alpha \in(0, \bar{\alpha}] .
$$


para todo $j \in I_{\varepsilon}\left(x_{*}\right)$. (5.7).

Em particular, para todo $j \in\{1, \ldots, m\}$ tal que $f_{j}\left(x_{*}\right)=f\left(x_{*}\right)$, vale

Defina

$$
\begin{aligned}
& \varepsilon_{1}=\min _{j: f_{j}\left(x_{*}\right)<f\left(x_{*}\right)}\left\{f\left(x_{*}\right)-f_{j}\left(x_{*}\right)\right\} \in(0, \infty], \\
& \varepsilon_{2}=\min _{j: f_{j}\left(x_{*}\right)>f\left(x_{*}\right)}\left\{f_{j}\left(x_{*}\right)-f\left(x_{*}\right)\right\} \in(0, \infty],
\end{aligned}
$$

Seja $\tilde{\alpha} \leq \bar{\alpha}$ tal que

$$
\left|f_{j}\left(x_{*}+\alpha d\right)-f_{j}\left(x_{*}\right)\right|<\frac{\min \left\{\varepsilon_{1}, \varepsilon_{2}\right\}}{2}
$$

para todo $j=1, \ldots, m, \alpha \in(0, \tilde{\alpha}]$.

Portanto, para todo $\alpha \in(0, \tilde{\alpha}]$, um índice $j$ tal que

$$
f\left(x_{*}+\alpha d\right)=f_{j}\left(x_{*}+\alpha d\right)
$$

é um dos índices $j$ tal que $f\left(x_{*}\right)=f_{j}\left(x_{*}\right)$. Em outras palavras, este índice pertence a $I_{0}\left(x_{*}\right) \subset I_{\varepsilon}\left(x_{*}\right)$. Mas, por (5.7),

$$
f_{j}\left(x_{*}+\alpha d\right)<f_{j}\left(x_{*}\right)
$$

para todo $\alpha \in(0, \tilde{\alpha}]$. Logo, $x_{*}$ não é um minimizador local.

Descrevemos abaixo o algoritmo principal. Ele é chamado de método primal porque somente as variáveis primais $x$ são manipuladas e atualizadas a cada iteração. $\mathrm{O}$ algoritmo encontra uma sequência decrescente de valores funcionais usando direções originárias da solução (inexata) de um problema de programação convexa.

\section{Algoritmo 2.1}

Seja $x_{0} \in \Omega$ um ponto inicial arbitrário. Seja $\theta \in(0,1), \Delta>0, \varepsilon>0$, $0<\sigma_{\min }<\sigma_{\max }<1, \eta \in(0,1]$.

Dado $x_{k} \in \Omega$ os passos da $k$-ésima iteração são:

Passo 1. (Resolvendo o subproblema)

Defina

$$
M_{k}(d)=\max _{j \in I_{\varepsilon}\left(x_{k}\right)} g_{j}\left(x_{k}\right)^{T} d .
$$

Considere o subproblema

$$
\text { Minimizar }_{d} M_{k}(d) \text { s.a. } x_{k}+d \in \Omega,\|d\|_{\infty} \leq \Delta .
$$


Observe que (5.9) é equivalente ao problema de otimização convexo

$$
\begin{gathered}
\text { Minimizar } w \\
g_{j}\left(x_{k}\right)^{T} d \leq w \forall j \in I_{\varepsilon}\left(x_{k}\right), \\
x_{k}+d \in \Omega, \quad\|d\|_{\infty} \leq \Delta .
\end{gathered}
$$

Seja $\bar{d}_{k}$ a solução de (5.9). (Veremos depois que não precisaremos calculálo.) Seja $d_{k}$ tal que $x_{k}+d_{k} \in \Omega,\left\|d_{k}\right\| \leq \Delta$ e

$$
M_{k}\left(d_{k}\right) \leq \eta M_{k}\left(\bar{d}_{k}\right) .
$$

Se $M_{k}\left(d_{k}\right)=0$ pare.

Passo 2. (Calculo do tamanho de passo)

Seja $\alpha \leftarrow 1$.

$\mathrm{Se}$

$$
f\left(x_{k}+\alpha d_{k}\right) \leq f\left(x_{k}\right)+\theta \alpha M_{k}\left(d_{k}\right)
$$

defina $\alpha_{k}=\alpha, x_{k+1}=x_{k}+\alpha_{k} d_{k}$ e termine a iteração. Senão, escolha $\alpha_{\text {new }} \in\left[\sigma_{\min } \alpha, \sigma_{\max } \alpha\right]$, defina $\alpha \leftarrow \alpha_{\text {new }}$ e repita o teste (5.11).

O lema a seguir é um lema técnico que será útil na prova de convergencia.

Lema 5.2.2. Suponha que $a_{1}, \ldots, a_{r}$ são números reais tais que

$$
a_{1} \leq a_{2} \leq \ldots \leq a_{q} \leq \ldots \leq a_{r} .
$$

Suponha que $\beta>0$ e $b_{1}, \ldots, b_{r} \in \mathbb{R}$ satisfaçam

$$
b_{j} \leq a_{j}-\beta \forall j=1, \ldots, r
$$

$e$

$$
b_{i_{1}} \leq b_{i_{2}} \leq \ldots \leq b_{i_{q}} \leq \ldots \leq b_{i_{r}} .
$$

Então,

$$
b_{i_{q}} \leq a_{q}-\beta \text {. }
$$

Prova. Claramente,

$$
b_{i_{q}} \leq a_{i_{q}}-\beta, b_{i_{q}} \leq b_{i_{q+1}} \leq a_{i_{q+1}}-\beta, \ldots, b_{i_{q}} \leq b_{i_{r}} \leq a_{i_{r}}-\beta .
$$

Portanto,

$$
b_{i_{q}} \leq \min \left\{a_{i_{q+1}}, \ldots, a_{i_{r}}\right\}-\beta \text {. }
$$


Mas, como $a_{1} \leq \ldots \leq a_{q} \leq \ldots \leq a_{r}$, segue que

$$
\min \left\{a_{i_{q+1}}, \ldots, a_{i_{r}}\right\} \leq a_{q} .
$$

Logo,

$$
b_{i_{q}} \leq a_{q}-\beta
$$

como queríamos provar.

No próximo teorema estabeleceremos que, se o iterado $x_{k}$ não for $\varepsilon$ ótimo, então $x_{k+1}$ está bem definido e $\alpha_{k}$ é limitado, e diferente de zero.

Teorema 5.2.3. Suponha que $x_{k} \in \Omega$ é o k-ésimo iterado do Algoritmo 2.1. Então:

(a) O algoritmo pára em $x_{k}$ se, e somente se, $x_{k}$ for $\varepsilon$-ótimo.

(b) Se o algoritmo não parar em $x_{k}$, então a iteração está bem definida $e$

$$
\alpha_{k} \geq \min \left\{\frac{2 \sigma_{\min } \gamma_{k}(1-\theta)}{L \Delta^{2}}, \frac{\varepsilon \sigma_{\min }}{3 c \Delta}\right\}
$$

onde

$$
\gamma_{k}=-\max _{j \in I_{\varepsilon}\left(x_{k}\right)}\left\{g_{j}\left(x_{k}\right)^{T} d_{k}\right\}>0 .
$$

Prova. Se o algoritmo parar em $x_{k}$, então $M_{k}\left(d_{k}\right)=0$. Portanto, por (5.10), $M_{k}\left(\bar{d}_{k}\right)=0$. Com isso, $M_{k}(d) \geq 0$ para todo $d \in \mathcal{D}$ tal que $\|d\|_{\infty} \leq \Delta$. Assim, $M_{k}(d) \geq 0$ para todo $d \in \mathcal{D}$. Isto implica que $x_{k}$ é $\varepsilon$-otimo.

Reciprocamente, se $x_{k}$ for $\varepsilon$-otimo, é necessário que $M_{k}\left(\bar{d}_{k}\right)=0$, e então $M_{k}\left(d_{k}\right)=0$ e o algoritmo pára em $x_{k}$.

Se o algoritmo não parar em $x_{k}$, então $M_{k}\left(d_{k}\right)<0$. Logo,

$$
-\gamma_{k}=\max _{j \in I_{\varepsilon}\left(x_{k}\right)}\left\{g_{j}\left(x_{k}\right)^{T} d_{k}\right\}<0 .
$$

Suponha que

$$
\alpha \in\left[0, \frac{2 \gamma_{k}(1-\theta)}{L \Delta^{2}}\right]
$$

Então,

$$
\frac{L \alpha \Delta^{2}}{2} \leq(1-\theta) \gamma_{k}
$$

Assim,

$$
\frac{L \alpha \Delta^{2}}{2} \leq(\theta-1) g_{j}\left(x_{k}\right)^{T} d_{k} \quad \forall j \in I_{\varepsilon}\left(x_{k}\right)
$$


e com isso

$$
g_{j}\left(x_{k}\right)^{T} d_{k}+\frac{L \alpha \Delta^{2}}{2} \leq \theta g_{j}\left(x_{k}\right)^{T} d_{k} \forall j \in I_{\varepsilon}\left(x_{k}\right)
$$

Portanto, como $\left\|\alpha d_{k}\right\|_{\infty} \leq \Delta$,

$$
\alpha g_{j}\left(x_{k}\right)^{T} d_{k}+\frac{L \alpha^{2}\left\|d_{k}\right\|_{\infty}^{2}}{2} \leq \alpha \theta g_{j}\left(x_{k}\right)^{T} d_{k} \quad \forall j \in I_{\varepsilon}\left(x_{k}\right) .
$$

E assim segue que

$$
f_{j}\left(x_{k}\right)+g_{j}\left(x_{k}\right)^{T}\left(\alpha d_{k}\right)+\frac{L}{2}\left\|\alpha d_{k}\right\|_{\infty}^{2} \leq f_{j}\left(x_{k}\right)+\alpha \theta g_{j}\left(x_{k}\right)^{T} d_{k} \quad \forall j \in I_{\varepsilon}\left(x_{k}\right) .
$$

Logo, por (5.4)

$$
f_{j}\left(x_{k}+\alpha d_{k}\right) \leq f_{j}\left(x_{k}\right)+\alpha \theta g_{j}\left(x_{k}\right)^{T} d_{k} \forall j \in I_{\varepsilon}\left(x_{k}\right) .
$$

Com isso provamos que, se $\alpha \in\left[0,2 \gamma_{k}(1-\theta) /\left(L \Delta^{2}\right)\right]$,

$$
f_{j}\left(x_{k}+\alpha d_{k}\right) \leq f_{j}\left(x_{k}\right)+\alpha \theta M_{k}\left(d_{k}\right) \forall j \in I_{\varepsilon}\left(x_{k}\right) .
$$

Por outro lado, se $\alpha \in[0, \varepsilon /(3 c \Delta)]$, segue que $\alpha c \Delta \leq \varepsilon / 3$, e assim $\alpha c\left\|d_{k}\right\|_{\infty} \leq \varepsilon / 3$, e $c\left\|\alpha d_{k}\right\|_{\infty} \leq \varepsilon / 3$, logo, by (5.3),

$$
\left|f_{j}\left(x_{k}+\alpha d_{k}\right)-f_{j}\left(x_{k}\right)\right| \leq \frac{\varepsilon}{3} \forall j=1, \ldots, m .
$$

Logo, para todo $\ell=1, \ldots, p$,

$$
f_{i_{\ell}\left(x_{k}\right)}\left(x_{k}+\alpha d_{k}\right) \leq f_{i_{\ell}\left(x_{k}\right)}\left(x_{k}\right)+\frac{\varepsilon}{3} \leq f\left(x_{k}\right)+\frac{\varepsilon}{3}
$$

e, para todo $\ell=p, \ldots, m$,

$$
f_{i_{\ell}\left(x_{k}\right)}\left(x_{k}+\alpha d_{k}\right) \geq f_{i_{\ell}\left(x_{k}\right)}\left(x_{k}\right)-\frac{\varepsilon}{3} \geq f\left(x_{k}\right)-\frac{\varepsilon}{3} .
$$

Isto significa que ao menos $p$ elementos do conjunto

$$
\left\{f_{1}\left(x_{k}+\alpha d_{k}\right), \ldots, f_{m}\left(x_{k}+\alpha d_{k}\right)\right\}
$$

são menores ou iguais a $f\left(x_{k}\right)+\varepsilon / 3$ e que ao menos $m-p+1$ elementos daquele conjunto são maiores ou iguais a $f\left(x_{k}\right)-\varepsilon / 3$.

Portanto,

$$
f\left(x_{k}+\alpha d_{k}\right)=f_{i_{p}\left(x_{k}+\alpha d_{k}\right)}\left(x_{k}+\alpha d_{k}\right) \in\left[f\left(x_{k}\right)-\frac{\varepsilon}{3}, f\left(x_{k}\right)+\frac{\varepsilon}{3}\right]
$$


Suponha que $j \notin I_{\varepsilon}\left(x_{k}\right)$. Então, ou $f_{j}\left(x_{k}\right)<f\left(x_{k}\right)-\varepsilon$ ou $f_{j}\left(x_{k}\right)>$ $f\left(x_{k}\right)+\varepsilon$. No primeiro caso, de (5.15) segue que

$$
f_{j}\left(x_{k}+\alpha d_{k}\right)<f\left(x_{k}\right)-\frac{2}{3} \varepsilon
$$

e assim, por (5.16),

$$
f_{j}\left(x_{k}+\alpha d_{k}\right)<f\left(x_{k}+\alpha d_{k}\right) .
$$

Analogamente, se $f_{j}\left(x_{k}\right)>f\left(x_{k}\right)+\varepsilon$, então

$$
f_{j}\left(x_{k}+\alpha d_{k}\right)>f\left(x_{k}+\alpha d_{k}\right) .
$$

Portanto,

$$
f\left(x_{k}+\alpha d_{k}\right)=f_{j}\left(x_{k}+\alpha d_{k}\right) \text { for some } j \in I_{\varepsilon}\left(x_{k}\right) .
$$

Escreva

$$
I_{\varepsilon}\left(x_{k}\right)=\left\{j_{1}, \ldots, j_{\nu}\right\}=\left\{j_{1}^{\prime}, \ldots, j_{\nu}^{\prime}\right\}
$$

onde

$$
f_{j_{1}}\left(x_{k}\right) \leq \ldots \leq f_{j_{\nu}}\left(x_{k}\right)
$$

e

$$
f_{j_{1}^{\prime}}\left(x_{k}+\alpha d_{k}\right) \leq \ldots \leq f_{j_{\nu}^{\prime}}\left(x_{k}+\alpha d_{k}\right) .
$$

Claramente, existe $q \in\{1, \ldots, \nu\}$ tal que

$$
i_{p}\left(x_{k}\right)=j_{q} .
$$

Agora, os índices $j \notin I_{\varepsilon}\left(x_{k}\right)$ tais que $f_{j}\left(x_{k}\right)<f\left(x_{k}\right)$ são os mesmos índices $j \notin I_{\varepsilon}\left(x_{k}\right)$ tais que $f_{j}\left(x_{k}+\alpha d_{k}\right)<f\left(x_{k}\right)$ e, mais ainda, os índices $j \notin$ $I_{\varepsilon}\left(x_{k}\right)$ tais que $f_{j}\left(x_{k}\right)>f\left(x_{k}\right)$ são os mesmos índices $j \notin I_{\varepsilon}\left(x_{k}\right)$ tais que $f_{j}\left(x_{k}+\alpha d_{k}\right)>f\left(x_{k}\right)$. Assim,

$$
i_{p}\left(x_{k}+\alpha d_{k}\right)=j_{q}^{\prime} .
$$

Então, de (5.14) e do Lemma 5.2.2, segue que, quando

$$
\alpha \in\left[0, \min \left\{\frac{2 \gamma_{k}(1-\theta)}{L \Delta^{2}}, \frac{\varepsilon}{3 c \Delta}\right\}\right]
$$

temos:

$$
f_{j_{q}^{\prime}}\left(x_{k}+\alpha d_{k}\right) \leq f_{j_{q}}\left(x_{k}\right)+\alpha \theta M_{k}\left(d_{k}\right) .
$$


Portanto,

$$
f_{i_{p}\left(x_{k}+\alpha d_{k}\right)}\left(x_{k}+\alpha d_{k}\right) \leq f_{i_{p}\left(x_{k}\right)}\left(x_{k}\right)+\alpha \theta M_{k}\left(d_{k}\right)
$$

Assim,

$$
f\left(x_{k}+\alpha d_{k}\right) \leq f\left(x_{k}\right)+\alpha \theta M_{k}\left(d_{k}\right)
$$

Portanto, quando (5.17) valer, o teste (5.11) também deve valer. Isto significa que $o$ valor de $\alpha$ que não satisfaz (5.11) não pode ser menor que $\min \left\{2 \gamma_{k}(1-\theta) /\left(L \Delta^{2}\right), \varepsilon /(3 c \Delta)\right\}$. Assim, o valor de $\alpha$ aceitável deve satisfazer:

$$
\alpha_{k} \geq \min \left\{\frac{2 \sigma_{\min } \gamma_{k}(1-\theta)}{L \Delta^{2}}, \frac{\varepsilon \sigma_{\min }}{3 c \Delta}\right\}
$$

como queríamos provar.

O principal resultado de convergência é dado pelo teorema 5.2.5. Antes disso, precisamos provar um simples lema preparatório.

Lema 5.2.4. Se $\left\{x_{k}\right\}$ é uma sequência infinita gerada pelo Algoritmo 2.1, então ou

$$
\lim _{k \rightarrow \infty} f\left(x_{k}\right)=-\infty
$$

ou

$$
\lim _{k \rightarrow \infty} M_{k}\left(d_{k}\right)=0 .
$$

Prova. Se (5.18) não vale, então, por (5.11), temos que

$$
\lim _{k \rightarrow \infty} \alpha_{k} M_{k}\left(d_{k}\right)=0 .
$$

Pelo Teorema 5.2.3, isto implica que vale (5.19).

Teorema 5.2.5. Suponha que $x_{*} \in \Omega$ é um ponto limite de uma sequência gerada pelo Algoritmo 2.1. Então $x_{*}$ é E-otimo.

Prova. Pelo fato de $f\left(x_{k+1}\right) \leq f\left(x_{k}\right)$ para todo $k=0,1,2, \ldots$ e $x_{*}$ ser um ponto limite de $\left\{x_{k}\right\}$ segue que $f\left(x_{k}\right) \rightarrow f\left(x_{*}\right)$. Portanto, pelo lema 5.2.4, (5.19) é válido. Seja $K$ uma sequência infinita de índices tal que

$$
\lim _{k \in K} x_{k}=x_{*} .
$$


Se $x_{*}$ não é $\varepsilon$-otima, então existe $\gamma>0$ e $d \in \mathbb{R}^{n}$ tais que $x_{*}+d \in \Omega$ e

$$
g_{j}\left(x_{*}\right)^{T} d \leq-\gamma \forall j \in I_{\varepsilon}\left(x_{*}\right) .
$$

Sem perda de generalidade, podemos assumir que $\|d\| \leq \Delta / 2$. Por continuidade, para $k$ grande o suficiente, $k \in K$, definindo

$$
\hat{d}_{k}=d+x_{*}-x_{k}
$$

temos que $\left\|\hat{d}_{k}\right\|_{\infty} \leq \Delta, x_{k}+\hat{d}_{k} \in \Omega \mathrm{e}$

$$
\lim _{k \in K} \hat{d}_{k}=d
$$

De (5.19), segue que $\lim _{k \rightarrow \infty} M_{k}\left(\bar{d}_{k}\right)=0$. Assim, $\liminf _{k \rightarrow \infty} M_{k}\left(\hat{d}_{k}\right) \geq$ 0 . Para todo $k \in K$ existe $j \in I_{\varepsilon}\left(x_{k}\right)$ tal que $g_{j}\left(x_{k}\right)^{T} \hat{d}_{k}=M_{k}\left(\hat{d}_{k}\right)$. Pelo fato de $I_{\varepsilon}\left(x_{k}\right)$ ser finita, existe $j$ tal que $g_{j}\left(x_{k}\right)^{T} \hat{d}_{k}=M_{k}\left(\hat{d}_{k}\right)$ infinitas vezes. Portanto, para aquele particular indice $j$,

$$
\liminf _{k \in K} g_{j}\left(x_{k}\right)^{T} \hat{d}_{k}=0 .
$$

Logo, tomando os limites,

$$
g_{j}\left(x_{*}\right)^{T} d=0 .
$$

Mas, para infinitos indices, desde que $j \in I_{\varepsilon}\left(x_{k}\right)$,

$$
f\left(x_{k}\right)-\varepsilon \leq f_{j}\left(x_{k}\right) \leq f\left(x_{k}\right)+\varepsilon,
$$

assim, tomando os limites,

$$
f\left(x_{*}\right)-\varepsilon \leq f_{j}\left(x_{*}\right) \leq f\left(x_{*}\right)+\varepsilon,
$$

e então $j \in I_{\mathcal{\varepsilon}}\left(x_{*}\right)$.

Portanto, (5.22) contradiz (5.20).

\subsection{Calculando uma solução aproximada para o subproblema}

Se $n$ é grande ou $I_{\varepsilon}\left(x_{k}\right)$ contém muitos elementos, achar a solução exata de (5.9) pode ser bastante caro. Nestes casos, uma solução aproximada $d_{k}$ que satisfaz (5.10) pode ser encontrada seguindo o procedimento descrito abaixo. Para simplificar a descrição assumiremos que $\operatorname{Int}(\Omega)$, o interior do 
conjunto convexo $\Omega$, é não vazio. Escolha $\eta^{\prime} \in(\eta, 1)$. Os sucessivos iterados $x_{k}$ pertencerão a $\operatorname{Int}(\Omega)$. Assumiremos que exista um procedimento que calcule uma sequência $\left\{s_{\nu}\right\}$, onde $s_{\nu} \in \mathbb{R}^{n}$, tal que $s_{\nu} \rightarrow \bar{d}_{k}$ e uma sequência de limitantes $c_{\nu}$ tais que $c_{\nu} \rightarrow M_{k}\left(\bar{d}_{k}\right) \mathrm{e}$

$$
c_{\nu} \leq M_{k}\left(\bar{d}_{k}\right)
$$

para todo $\nu=0,1,2, \ldots$

Defina, para todo $\nu=0,1,2, \ldots$,

$$
\lambda_{\nu}=\min \left\{1, \max \left\{\lambda \geq 0 \mid x_{k}+\lambda s_{\nu} \in \Omega,\left\|\lambda s_{\nu}\right\|_{\infty} \leq \Delta\right\}\right\} .
$$

Como $\Omega$ é convexo e $x_{k}$ é interior, segue que

$$
\lambda_{\nu} \rightarrow 1,
$$

assim,

$$
x_{k}+\lambda_{\nu} s_{\nu} \rightarrow x_{k}+\bar{d}_{k}
$$

e

$$
M_{k}\left(\lambda_{\nu} s_{\nu}\right) \rightarrow M_{k}\left(\bar{d}_{k}\right) .
$$

Portanto, tomando $\nu$ grande o suficiente, obtemos $\lambda_{\nu}$ tal que

$$
M_{k}\left(\lambda_{\nu} s_{\nu}\right) \leq \eta^{\prime} c_{\nu} \leq \eta^{\prime} M_{k}\left(\bar{d}_{k}\right)
$$

Então,

$$
M_{k}\left(\frac{\eta}{\eta^{\prime}} \lambda_{\nu} s_{\nu}\right) \leq \eta M_{k}\left(\bar{d}_{k}\right)
$$

Isto implica que, tomando

$$
d_{k}=\frac{\eta}{\eta^{\prime}} \lambda_{\nu} s_{\nu}
$$

a condição (5.10) é satisfeita, $x_{k}+\alpha d_{k}$ continua interior para todo $\alpha \in[0,1]$. Assim, $x_{k+1}$ é interior e o processo pode ser repetido na próxima iteração. 


\section{Capítulo 6}

\section{Experimentos Numéricos}

Neste capítulo nosso intuito é utilizar a reformulação do problema OVO para resolver um problema de ajustamento de parâmetros linear. Faremos uma comparação das abordagens do problema, a saber:

Abordagem MPEC uso de algoritmos com convergência AGP, como sugerido no capítulo 2. Para isso usaremos MILONGA, que é um método baseado em lagrangeano aumentado que resolve subproblemas com restrições em caixa usando estratégia global, descrita em [4], e direções de Newton dentro das faces.

Abordagem Cauchy-Primal uso do algoritmo primal de Cauchy, desenvolvido no capítulo 5 .

PROBLEMA: Queremos ajustar o modelo

$$
y(t, x)=x_{1}+x_{2} t+x_{3} t^{2}+x_{4} t^{3}
$$

a um conjunto de dados $\left(t_{i}, y_{i}\right), i=1, \ldots, m$. Estes dados foram gerados a partir da solução pré-fixada

$$
x^{*}=\left(x_{1}^{*}, x_{2}^{*}, x_{3}^{*}, x_{4}^{*}\right)=(0,2,-3,1),
$$

fazendo

$$
\begin{gathered}
w_{i}=y\left(t_{i}, x^{*}\right), \\
t_{i}=-1+0.1 i, \quad i=0, \ldots, m, m=46, \\
y_{i}=10 \text { if } i=7, \ldots, 16, \\
y_{i}=w_{i}+r_{i}, \text { caso contrário, }
\end{gathered}
$$


onde $r_{i}$ é um número gerado aleatoriamente entre -0.01 e 0.01 . Portanto, $y_{7}, \ldots, y_{16}$ simulam observaçãoes com erros, ou outliers. Na Tabela 6.1 temos os dados $\left(t_{i}, y_{i}\right)$.

\begin{tabular}{|c|c|c||c|c|c||c|c|c|}
\hline$i$ & $t_{i}$ & $y_{i}$ & $i$ & $t_{i}$ & $y_{i}$ & $i$ & $t_{i}$ & $y_{i}$ \\
\hline 1 & -1.0000 & -5.8000 & 17 & 0.6000 & 0.5360 & 33 & 2.2000 & 0.3280 \\
\hline 2 & -0.9000 & -5.1590 & 18 & 0.7000 & 0.4730 & 34 & 2.3000 & 1.0970 \\
\hline 3 & -0.8000 & -4.2320 & 19 & 0.8000 & -0.0080 & 35 & 2.4000 & 1.1440 \\
\hline 4 & -0.7000 & -3.4130 & 20 & 0.9000 & 0.2990 & 36 & 2.5000 & 1.6750 \\
\hline 5 & -0.6000 & -2.6960 & 21 & 1.0000 & 0.2000 & 37 & 2.6000 & 2.2960 \\
\hline 6 & -0.5000 & -1.6750 & 22 & 1.1000 & -0.2990 & 38 & 2.7000 & 3.4130 \\
\hline 7 & -0.4000 & 10.0000 & 23 & 1.2000 & 0.0080 & 39 & 2.8000 & 4.2320 \\
\hline 8 & -0.3000 & 10.0000 & 24 & 1.3000 & -0.0730 & 40 & 2.9000 & 5.1590 \\
\hline 9 & -0.2000 & 10.0000 & 25 & 1.4000 & -0.5360 & 41 & 3.0000 & 6.2000 \\
\hline 10 & -0.1000 & 10.0000 & 26 & 1.5000 & -0.5750 & 42 & 3.1000 & 6.9610 \\
\hline 11 & 0 & 10.0000 & 27 & 1.6000 & -0.5840 & 43 & 3.2000 & 8.6480 \\
\hline 12 & 0.1000 & 10.0000 & 28 & 1.7000 & -0.5570 & 44 & 3.3000 & 9.6670 \\
\hline 13 & 0.2000 & 10.0000 & 29 & 1.8000 & -0.4880 & 45 & 3.4000 & 11.2240 \\
\hline 14 & 0.3000 & 10.0000 & 30 & 1.9000 & 0.0290 & 46 & 3.5000 & 12.9250 \\
\hline 15 & 0.4000 & 10.0000 & 31 & 2.0000 & -0.2000 & & & \\
\hline 16 & 0.5000 & 10.0000 & 32 & 2.1000 & 0.0310 & & & \\
\hline
\end{tabular}

Tabela 6.1: Dados para o ajustamento linear com 4 parametros.

A seguir descrevemos o resultado de cada método usado, destacando os coeficientes fixados nos algoritmos.

Abordagem MPEC com Milonga O problema a ser resolvido é dado por

$$
\text { OVO }^{*}\left\{\begin{array}{l}
\text { Minimizar } z \\
\text { Sujeito a } \\
\sum_{i=1}^{m} r_{i} w_{i}=0 \\
\sum_{i=1}^{m}\left(1-r_{i}\right) u_{i}=0 \\
\sum_{i=1}^{m} r_{i}=p \\
u_{i}-z+f_{i}(x)-w_{i}=0, \quad i=1, \ldots, m \\
u \geq 0, \quad 0 \leq r \leq e w \geq 0, x \in \Omega .
\end{array}\right.
$$


onde

$$
\begin{gathered}
m=46 \\
f_{i}(x)=\left(x_{1}+x_{2} t_{i}+x_{3} t_{i}^{2}+x_{4} t_{i}^{3}-y_{i}\right)^{2}, \quad i=1, \ldots, m .
\end{gathered}
$$

Foi usado o ponto inicial $x_{0}=(0,0,0,0)$, para todos os valores de $p$ utilizados. As soluções obtidas estão na tabela 6.2.

\begin{tabular}{|c|c|c|c|c|c|}
\hline$p$ & $x_{1}$ & $x_{2}$ & $x_{3}$ & $x_{4}$ & $f o b j$ \\
\hline 20 & 0.6352 & 1.5879 & -3.1312 & 1.0552 & $2.3315 \mathrm{D}-02$ \\
\hline 22 & 0.1665 & 2.1209 & -3.3314 & 1.0975 & $2.3595 \mathrm{D}-02$ \\
\hline 24 & 0.3569 & 2.0948 & -3.3967 & 1.0968 & $3.1558 \mathrm{D}-02$ \\
\hline 26 & 0.2769 & 2.1731 & -3.4527 & 1.1278 & $3.3293 \mathrm{D}-02$ \\
\hline 28 & $1.5487 \mathrm{D}-04$ & 2.0001 & -3.0002 & 1.0000 & $3.9994 \mathrm{D}-02$ \\
\hline 30 & $5.1636 \mathrm{D}-05$ & 2.0000 & -3.0001 & 1.0000 & $3.9994 \mathrm{D}-02$ \\
\hline 32 & 7.2887 & 0.8514 & -7.0558 & 2.1486 & 9.1984 \\
\hline 34 & $8.9409 \mathrm{D}-06$ & 2.0000 & -3.0000 & 1.0000 & $3.9998 \mathrm{D}-02$ \\
\hline 36 & $2.1265 \mathrm{D}-05$ & 2.0001 & -3.0001 & 1.0000 & $3.0204 \mathrm{D}-05$ \\
\hline 38 & 5.8950 & 1.8337 & -7.9721 & 2.3878 & 16.8511 \\
\hline 40 & 5.7799 & 2.0595 & -7.0716 & 2.1214 & 19.2898 \\
\hline
\end{tabular}

Tabela 6.2: Solução do Milonga para o ajuste linear de 4 parâmetros.

Os resultados são coerentes com o que se esperava obter, pois a solução real do problema, $x^{*}(0,2,-3,1)$ foi alcançada para valores do parâmetro $p$ próximos do seu valor ideal $p=36$, já que sabemos de antemão o número de dados corrompidos da amostra deste exemplo. Para $p=32$ o algoritmo nos forneceu um minimizador local, e para $p>36$ os dados corrompidos levaram a respostas completamente erradas. Isto se deve ao fato de a formulação OVO $\operatorname{com} p>36$ contabilizar estes dados ruins como se fossem dados corretos da amostra.

Método Cauchy Primal Utilizamos o Algoritmo 2.1, com os parâmetros fixados

$$
\Delta=1.0, \quad \theta=0.5, \quad \sigma_{\min }=0.1, \quad \sigma_{\max }=0.9, \quad \eta=1.0, \quad \varepsilon=0.001 .
$$

e os limitantes

$$
-10 \leq x_{i} \leq 10, \quad i=1,2,3,4 .
$$

As soluções obtidas pelo algoritmo para diferentes valores de $p$ são mostradas na Tabela 6.3 , onde $p$ define a função ordem-p, n.iter é o 
número de iterações, fobj é o valor da função objetivo. Observe que o "correto" $p$ deveria ser 36 , porque geramos 10 outliers.

\begin{tabular}{|c|c|c|c|c|c|c|}
\hline$p$ & $x_{1}$ & $x_{2}$ & $x_{3}$ & $x_{4}$ & fobj & $n$. iter \\
\hline 20 & -0.0060 & 1.9892 & -2.9568 & 0.9748 & 0.0404 & 33 \\
\hline 21 & -0.1231 & 1.2809 & -2.4353 & 0.8965 & 0.0369 & 47 \\
\hline 22 & 0.3957 & 2.3052 & -3.6787 & 1.1742 & 0.0297 & 85 \\
\hline 23 & 0.1116 & 1.8346 & -2.9223 & 0.9883 & 0.0401 & 49 \\
\hline 24 & -0.0081 & 2.0035 & -2.9897 & 0.9943 & 0.0405 & 41 \\
\hline 25 & 0.1033 & 1.8469 & -2.9291 & 0.9896 & 0.0402 & 67 \\
\hline 26 & 0.0261 & 1.9557 & -2.9762 & 0.9958 & 0.0403 & 70 \\
\hline 27 & 9.0443 & -7.4778 & -1.4899 & 1.2465 & 2.9491 & 78 \\
\hline 28 & -0.0190 & 2.0336 & -3.0177 & 1.0028 & 0.0405 & 49 \\
\hline 29 & 0.0015 & 1.9917 & -2.9939 & 0.9989 & 0.0407 & 52 \\
\hline 30 & 0.0096 & 1.9841 & -2.9919 & 0.9987 & 0.0403 & 68 \\
\hline 31 & 0.0030 & 2.0024 & -3.0044 & 1.0011 & 0.0407 & 57 \\
\hline 32 & 0.0001 & 2.0030 & -3.0010 & 0.9999 & 0.0408 & 66 \\
\hline 33 & 0.0021 & 2.0007 & -3.0024 & 1.0007 & 0.0407 & 49 \\
\hline 34 & 7.8217 & 5.8390 & -10.0000 & 2.4904 & 14.1827 & 158 \\
\hline 35 & -0.0003 & 2.0004 & -2.9997 & 0.9999 & 0.0403 & 56 \\
\hline 36 & 0.0000 & 2.0003 & -3.0002 & 1.0000 & 0.0403 & 67 \\
\hline 37 & 7.3236 & 1.6767 & -8.3264 & 2.4949 & 13.0175 & 187 \\
\hline 38 & 9.6125 & -9.6561 & -0.5977 & 1.1134 & 10.9439 & 138 \\
\hline 39 & 10.0000 & -6.4565 & -3.4642 & 1.6757 & 15.0919 & 130 \\
\hline 40 & 6.2406 & 2.2166 & -8.3576 & 2.4577 & 16.5412 & 295 \\
\hline 41 & 6.2025 & 2.5359 & -8.6241 & 2.5104 & 17.1385 & 283 \\
\hline 42 & 6.3137 & 1.7981 & -8.3196 & 2.4870 & 19.3482 & 207 \\
\hline 43 & 7.7660 & 2.3983 & -9.6980 & 2.8103 & 24.2539 & 152 \\
\hline
\end{tabular}

Tabela 6.3: Solução OVO-Cauchy para o ajustamento de 4 parametros.

Os resultados se mostraram coerentes e satisfatórios. Como era esperado, para $p>36$ a solução dada pelo algoritmo está distante da solução $x_{*}$, com valor da função objetivo (OVO function) muito grande. Para $p=27$ e $p=34$ a solução obtida não foi um minimizador global da função OVO. Isto não é surpreendente, já que o algoritmo oferece somente pontos estacionários como solução, e portanto podendo gerar pontos críticos locais em alguns casos. Para todos os outros valores testados de $p$ a função objetivo esteve abaixo do valor $0.05 \mathrm{e}$ 
a solução obtida esteve próxima de $x_{*}$. O exemplo sugere que no caso de problemas reais, onde não se conhece o número de dados corrompidos, ou outliers, diferentes valores de $p$ deveriam ser testados para diferentes pontos iniciais e aceitar uma solução levando em conta os valores de $p$ da função OVO. 


\section{Capítulo 7}

\section{Conclusões}

Introduzimos o problema de otimização OVO, um problema contínuo, não diferenciável e, em geral, não convexo. O problema se aplica diretamente a modelos de ajustamento de parâmetros e, promissoramente, a tomada de decisão. A tentativa de se obter um algoritmo primal, cuja implementção baseia-se na solução ( aproximada) de subproblemas de Programação Linear, foi bem sucedida, gerando resultados computacionais satisfatórios nos problemas propostos.

O algoritmo OVO deu indícios de ser uma boa ferramenta para lidar com problemas de ajustamento robusto de parâmetros. Muitos esquemas mais eficientes de regressão, menos sensíveis a dados com erros do que algoritmos baseados em quadrados mínimos, foram introduzidos na literatura. Veja, por exemplo, [8], [9], [10] e referências da área. Uma abordagem usual é ponderar as diferenças dos quadrados por meio de um filtro que penalize "outliers". Esta metodologia requer algum conhecimento prévio sobre os dados que porventura devessem ser penalizados. Dessa forma o algoritmo OVO poderia ter um papel de filtro universal, no sentido de que o usuário não necessita saber de antemão qualquer informação qualitativa sobre os outliers, mas somente informar ao algoritmo um estimativa de sua cardinalidade na amostra original. A calibração do modelo dependeria somente de um único parâmetro unidimensional, sendo a penalização dos outliers feita internamente por OVO. Por outro lado, a abordagem OVO pode também ser usada para detectar outliers, deixando o procedimento de estimação para outro algoritmo, após ter detectado dados discrepantes.

Finalmente, o problema OVO parece ser um problema de otimização que generaliza a otimização com hipóteses de diferenciabilidade. Um campo promissor de pesquisa é adaptar algoritmos clássicos de otimização (veja, por 
exemplo, [6]) para o problema OVO. O algoritmo apresentado no capítulo 5 é, neste sentido, uma generalização do metodo steepest descent, que, como é bem sabido, é o mais clássico dos algoritmos para otimização. 


\section{Referências Bibliográficas}

[1] R. Andreani, C. Dunder e J. M. Martínez, Order-Value Optimization: Formulation and Solution by means of a Primal Cauchy Method, 2002.

[2] R. Andreani, J. M. Martínez e B. F. Svaiter. On the regularization of mixed complementarity problems. Numerical Functional Analysis and Optimization 21, pp. 589-600 (2000).

[3] R. Andreani e J. M. Martínez [2001], On the solution of bounded and unbounded mixed complementarity problems, to appear in Optimization.

[4] E. G. Birgin, J. M. Martínez, Large-scale active-set box-constrained optimization method with spectral projected gradients, Computational Optimization and Applications. 23, pp. 101-125 (2002).

[5] R. W. Cottle, J-S. Pang e R. E. Stone [1992], The linear complementarity problem, Academic Press, Boston.

[6] R. Fletcher, Practical Methods of Optimization, New York: Wiley, 1988.

[7] P. Hartman e G. Stampacchia. On some nonlinear elliptic differential functional equations, Acta Math. 115, 153-188 (1966).

[8] P. W. Holland e R. E. Welsch, Robust regression using iteratively reweighted least-squares, Communications in Statistics: Theory and Methods, A6, 813-827, 1977.

[9] M. A. Howe e B. Rustem. A robust hedging algorithm. Journal of Economic Dynamics and Control 21 (1997) 1065-1092.

[10] P. J. Huber, Robust Statistics, New York: Wiley, 1981. 
[11] O. L. Mangasarian, Nonlinear Programming, SIAMIS Classics in Applied Mathematics, SIAM Philadelphia, 1994.

[12] J. M. Martínez e B. F. Svaiter, A Practical optimality condition without constraint qualifications for nonlinear programming .

[13] M. Rom e M. Avriel, Properties of the Sequential GradientRestoration Algorithm (SGRA), Part 1: Introduction and Comparison with Related Methods, Journal of Optimization Theory and Applications, Vol. 62, pp. 77-98, 1989.

[14] M. Rom e M. Avriel, Properties of the Sequential GradientRestoration Algorithm (SGRA), Part 2: Convergence Analysis, Journal of Optimization Theory and Applications, Vol. 62, pp. 99-126, 1989. 